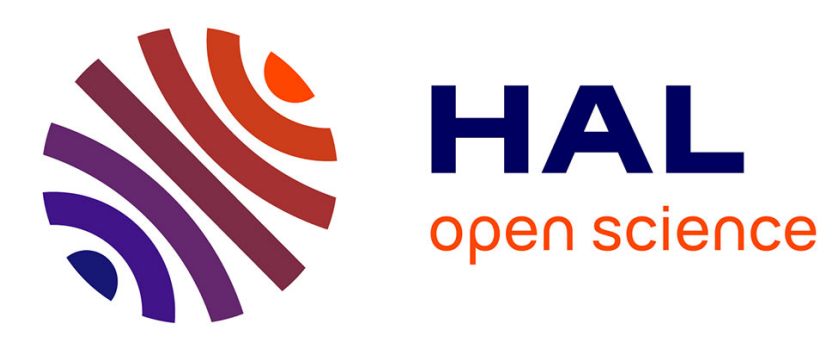

\title{
A silicon-position dependent 6-endo-trig cyclization during Tsuji-Trost alkylation
}

Jyoti Agarwal, Claude Commandeur, Max Malacria, Serge Thorimbert

\section{To cite this version:}

Jyoti Agarwal, Claude Commandeur, Max Malacria, Serge Thorimbert. A silicon-position dependent 6-endo-trig cyclization during Tsuji-Trost alkylation. Tetrahedron, 2013, 69 (45), pp.9398-9405. 10.1016/j.tet.2013.08.080 . hal-01196779

\section{HAL Id: hal-01196779 \\ https://hal.sorbonne-universite.fr/hal-01196779}

Submitted on 10 Sep 2015

HAL is a multi-disciplinary open access archive for the deposit and dissemination of scientific research documents, whether they are published or not. The documents may come from teaching and research institutions in France or abroad, or from public or private research centers.
L'archive ouverte pluridisciplinaire HAL, est destinée au dépôt et à la diffusion de documents scientifiques de niveau recherche, publiés ou non, émanant des établissements d'enseignement et de recherche français ou étrangers, des laboratoires publics ou privés. 


\title{
A silicon-position dependent 6-endo-trig cyclization during Tsuji-Trost alkylation
}

\author{
Jyoti Agarwal $^{\mathrm{a}}$, Claude Commandeur ${ }^{\mathrm{a}}$, Max Malacria $^{\mathrm{a}}$ and Serge Thorimbert ${ }^{\mathrm{a}}{ }^{*}$ \\ ${ }^{a}$ UPMC Sorbonne Universités, Institut Parisien de Chimie Moléculaire, UMR CNRS 7201, 4 place Jussieu, 75005 Paris, France
}

\section{ARTICLE INFO}

Keywords:

Tsuji-Trost alkylation

Cyclization

Palladium

Regioselectivity

Silicon derivatives

\section{ABSTRACT}

Two silylated cyclohexenes products have been prepared by using a Tsuji-Trost palladiumcatalyzed cyclization. It involves the generation of a cationic $\pi$-allylic palladium complex bearing a triethyl silyl group on $\mathrm{C}-3$, which cyclizes via a 6-endo-trig process to afford the cyclohexene derivatives. It is also demonstrated that the position of the silyl group on the starting allylic substrate strongly influenced the reaction. It could favor either the production of the expected cyclohexenyl ring or a diene by an elimination that occurs on the silyl-substituted $\mathrm{C}-2 \pi$-allylic palladium complex.

\section{Introduction}

Palladium-catalyzed allylic alkylation represents one of the most versatile protocols in organic chemistry. ${ }^{[1]}$ After the pioneering work of Tsuji and Trost, ${ }^{[2]}$ it has been applied successfully to the synthesis of many biological active molecules, natural products and other important organic skeletons. ${ }^{[3]}$ This alkylation occurred via a $\pi$-allylic palladium complex intermediate generated from the oxidative addition of $\operatorname{Pd}(0)$ into allylic substrates. Steric effects usually govern the regioselectivity of this reaction, but electronic properties of the substituents also greatly control the nucleophilic attack. ${ }^{[4]}$ Along with various applications of this protocol, many reports on palladium-catalyzed cyclization have been published. ${ }^{[5]}$ As well exposed by J.E. Baldwin, cyclization processes are mostly governed by the created ring size, the hybridization of the attacked carbon as well as the inside or outside position of the break bond in comparison to the formed cycle. ${ }^{[6]}$ Thus, the angle of approach of the nucleophile on the $\pi$-allylic complex is indicative of the feasibility of the reaction. It has been reported that malonyl vinylepoxides, in the presence of palladium catalyst, led to cyclobutanols in place of cyclohexenols via a 4-exo-trig process. ${ }^{[7]}$ Ionic 6-endo-trig cyclizations in carbon skeleton remain a challenging area and methods to synthesize cyclohexenes are still required even after the development of ring closing metathesis reactions. ${ }^{[8]}$

Since the pioneering work of Hirao, it is admitted that the presence of a silyl groups usually direct the attack of the nucleophiles to the distal position (relative to the silicon atom) of the palladium $\pi$-allylic cationic complex leading to the corresponding vinyl silanes. ${ }^{[9]}$ Such a regioselectivity may be accounted for in terms of steric factors, charge distribution of the allyl complex, as well as stability of the newly formed olefin$\operatorname{Pd}(0)$ complex. We reported a very strong silicon effect that afforded highly chemo- and stereoselective palladium-catalyzed alkylations ${ }^{[10]}$ that we later applied in synthesis. ${ }^{[11]}$ This directing group has been used to force the 5-endo-trig processes vs the classical 3-exo-trig but was unsuccessful for the 7-endo-trig vs the 5-exo-trig ones. ${ }^{[12]}$ All above facts drew our attention to study the effect of the position of a silyl group to direct a 6-endo-trig cyclization through the palladium-catalyzed allylation process. Considering this, we envisaged the synthesis of silylated cyclohexene products $\mathbf{C}$ through the formation of the $\pi$-allylic intermediates $\mathbf{B}$ which in turn, could be generated from the reaction of allylic acetate or carbonate precursors $\mathbf{A}$ with $\operatorname{Pd}(0)$ catalyst (Scheme 1)
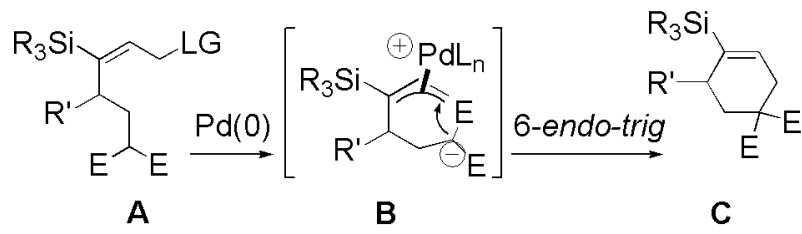

$E=$ electron withdrawing groups;
$L G=O A c, O \mathrm{CO}_{2} \mathrm{Me} ;$

$\mathrm{R}^{\prime}=\mathrm{H}, \mathrm{OH}$

Scheme 1. Silylated precursor A for 6-endo-trig cyclization.

\section{Results and Discussion}

We first considered the precursor $\mathbf{C}$ bearing one hydroxyl function on allylic position (Scheme $1 ; \mathrm{R}^{\prime}=\mathrm{OH}$ ). The expected resulting cyclohexene presents the correct functionalization for the synthesis of carbocycles of biological interest such as Shikimic acid for example.

We reasoned that the precursor 1 required for the cyclization could be prepared in few steps (hydrosilylation, acylation) via the alkyne resulting from the addition of the propargylic alcohol $\mathbf{3}$ to the malonyl aldehyde 2 (Scheme 2).<smiles>C=C(CC(OC)C(OC)OC)OCC=C([SiH2]CC)C(=O)OC</smiles>

Scheme 2. Approach towards the cyclization precursor 1. 
We observed degradation products during the condensation step between the malonyl aldehyde $\mathbf{2}$ and the propargylic alcohol derivative $\mathbf{3}$. It is noteworthy to mention here that the hydrogen atom present at $\alpha$-position related to the ester groups is acidic enough to give side reactions under the operating conditions. We thus masked this acidic proton by replacing it with a third ester function (Scheme 3).

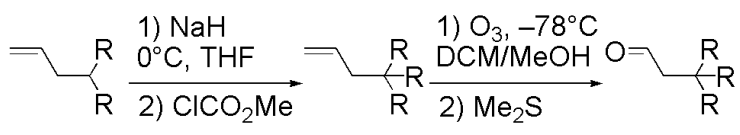

$$
\begin{aligned}
& \text { 4, } \mathrm{R}=\mathrm{CO}_{2} \mathrm{Me} \quad 5 \text {, quant. 6, quant. } \\
& 6+\mathrm{Li}=\mathrm{OTHP}_{3} \frac{\text { Additive }}{\mathrm{T}^{\circ}{ }^{\circ} \mathrm{C} \text { to rt }} \mathrm{R} \underset{\mathrm{R}}{\mathrm{THF}} \mathrm{OTHP}
\end{aligned}
$$

Additive : None, $\mathrm{CeCl}_{2}, \mathrm{CrCl}_{3}$

Scheme 3. Preparation of triester aldehyde $\mathbf{6}$ and synthesis of lactone 7 .

Thus, the allyl malonate $\mathbf{4}$ was converted into the triester $\mathbf{5}$ by alkylation with methyl chloroformate. ${ }^{[13]}$ The ozonolysis of the double bond delivered the expected aldehyde $\mathbf{6}$ in good yield over the two steps. Our next target was the preparation of the allylic acetate 1. For that purpose, we performed the alkylation of the aldehyde 6 with the lithiated anion of $O$-protected propargylic alcohol 3. The reaction was fast but the expected allylic alcohol was not observed. In its place, we isolated in nearly quantitative yield, the lactone 7 resulting from the intramolecular attack of the generated alkoxide ion to one of the ester functions. The more hindered triethyl ester also delivered the corresponding lactone. To avoid the formation of the lactone $\mathbf{7}$, we considered performing the alkylation in the presence of Cerium (III) or Chromium (III) chlorides or with the preformed ceric anion of $\mathbf{3}$. These conditions also led to the formation of the heterocyclic compound 7 with comparable yields.

Even if this lactone could be considered as a protection of the hydroxyl group, this ring should be open before the final cyclization step due to strong steric constraints. Moreover, both the THP protective group and the third ester should also be replaced. Starting from 7, we performed the simultaneous deprotection of the primary alcohol and the decarboxylation of one of the ester. Using a catalytic amount of $p$-TSA in $\mathrm{MeOH}$ at room temperature, we isolated the lactone $\mathbf{8}$ in $93 \%$ yield. Transesterification of $\mathbf{8}$ was then performed using $\mathrm{Ti}(\mathrm{O} i-\mathrm{Pr})_{4}$ in refluxing isopropanol. This protocol resulted in the formation of the corresponding diol 9 with $64 \%$ yield (Scheme 4 )

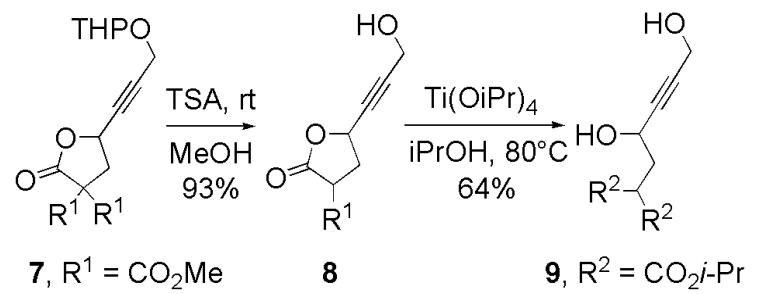

Scheme 4. Preparation of alkynyl diol 9

We then performed the hydrosilylation of the triple bond of the alkynyl diol 9 by using $\mathrm{Et}_{3} \mathrm{SiH}$ in presence of catalytic amount of $\mathrm{H}_{2} \mathrm{PtCl}_{6}$ (Figure 1). ${ }^{[14]}$ Surprisingly, when the reaction was performed in $i$-PrOH as solvent, no hydrosilylation was noticed after one night at $50^{\circ} \mathrm{C}$ (Table 1 , entry 1 ). In acetonitrile, only $20 \%$ of hydrosilylation product was obtained with the recovery of $70 \%$ of starting material (Table 1, entry 2). In neat conditions, the conversion was also not completed, affording mostly degradation products (Table 1 , entry 3 ). In all cases, no selectivity was apparent. Finally, the best result was obtained in THF, giving the corresponding regioisomers $\mathbf{1 0}$ and $\mathbf{1 1}$ in $90 \%$ yield in a 1:1 ratio. We also tested the direct hydrosilylation of the lactone 7 with tert-butyldimethylsilane, but this also led to a mixture of two isomers in a $59 \%$ yield.

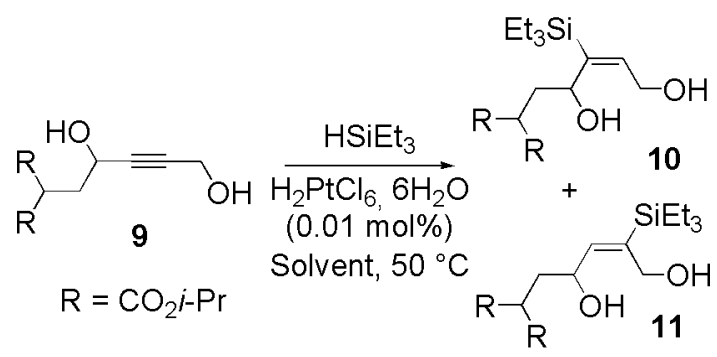

Figure 1. Hydrosilylation of 9.

Table 1. Solvent screening for the hydrosilylation of 9 .

\begin{tabular}{lllll}
\hline Entry & Solvent & Conversion $(\%)$ & Ratio 10:11 & Yield $(\%)^{\mathrm{a}}$ \\
\hline 1 & $i$-PrOH & - & - & - \\
2 & $\mathrm{CH}_{3} \mathrm{CN}$ & 30 & $1: 1$ & 20 \\
3 & Neat & 70 & $1: 1$ & 20 \\
4 & THF & 100 & $1: 1$ & 90 \\
\hline
\end{tabular}

${ }^{\mathrm{a}}$ Combined isolated yield of both regioisomers.

The two regioisomers $\mathbf{1 0}$ and $\mathbf{1 1}$ were separated by flash chromatography, and $\mathbf{1 0}$ was quantitatively converted into the corresponding allylic acetate $\mathbf{1 2}$ by using acetic anhydride and $\mathrm{NEt}_{3}$ in $\mathrm{CH}_{2} \mathrm{Cl}_{2}$. It should be noted that $\mathbf{1 0}, 11$ and 12 slowly cyclized into the corresponding more stable lactones (see experimental part for characterization). Therefore, it is essential to engage them relatively quickly after there preparation.

After the synthesis of the allylic acetate precursor 12, we performed the palladium-catalyzed 6-endo-trig cyclization. ${ }^{[15]}$ This reaction was carried out in presence of sodium hydride followed by the addition of $5 \mathrm{~mol} \%$ of $\mathrm{Pd}\left(\mathrm{PPh}_{3}\right)_{4}$ catalyst. The reaction worked well in $\mathrm{THF}$ at $65^{\circ} \mathrm{C}$ and we observed the consumption of the starting material in less than $3 \mathrm{~h}$. After purification, we did not observed the formation of the expected silylated cyclohexenol $\mathbf{1 3}$ but we isolated the conjugated trienic ester 15 in $42 \%$ yield. The ${ }^{1} \mathrm{H}$ and ${ }^{13} \mathrm{C}$ NMR indicated the presence of five vinylic protons and six vinylic carbons. HRMS definitively confirmed the proposed structure (Scheme 5).

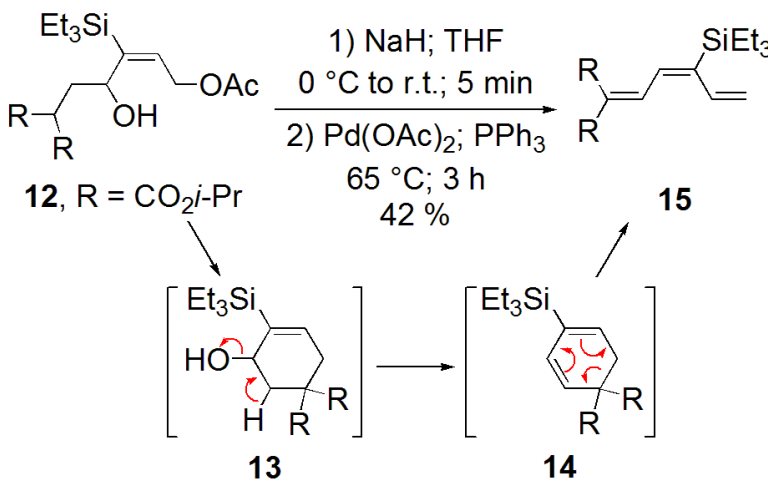

Scheme 5. Palladium-catalyzed cyclization of $\mathbf{1 2}$

We examined the formation of this compound, and hypothesized that the 6-endo-trig cyclization has taken place as expected. Indeed, we propose the formation of the desired 
cyclohexenol 13 followed by a dehydration reaction. The palladium complex could eventually mediate this undesired elimination. ${ }^{[16]}$ The resulting cyclohexadiene $\mathbf{1 4}$ then underwent a $6-\pi$ retro-electrocyclization giving the more stable conjugated triene $15 .{ }^{[17]}$ To validate the hypothesis that the formation of compound 15 occurred through a 6-endo-trig cyclization, and not merely a direct degradation of the precursor 12, we decided to synthesize a simplified precursor, which did not possesses a secondary hydroxyl function (Scheme 1, C; $\mathrm{R}^{\prime}=\mathrm{H}$ ). This model, required to validate our strategy, could be prepared by hydrosilylation of an alkyne precursor $\mathbf{1 6}$ previously reported by Deslongchamps (Table 3). ${ }^{[18]}$

The introduction of the triethylsilyl group onto $\mathbf{1 6}$ was conducted under standard $\mathrm{H}_{2} \mathrm{PtCl}_{6}$-catalyzed hydrosilylation. It delivered a mixture of two regioisomers $\mathbf{1 7}$ and $\mathbf{1 8}$ in a 2:3 ratio with an overall yield of $90 \%$ (Scheme 6). Few organometallic complexes were tested without real improvement. For example, when the rhodium dimer $[\mathrm{RhCl}(\operatorname{cod})]_{2}$ was used the yield decreased to $74 \%$ whereas the platine(II) precursor $\mathrm{PtCl}_{2}(\mathrm{PhCN})_{2}$ gave a slightly better $95 \%$ yield. The use of cationic ruthenium complexes mainly degradated the starting alkyne.

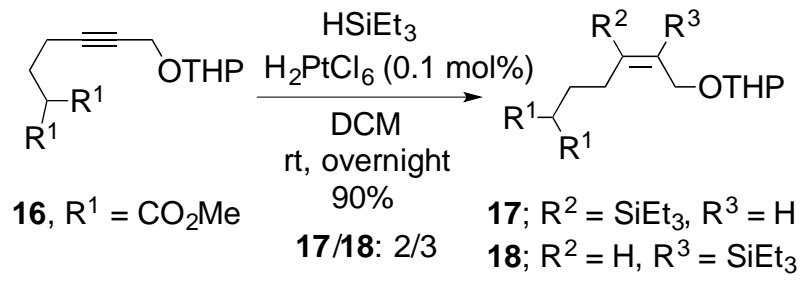

Scheme 6. Hydrosilylation of $\mathbf{1 6 .}$

The two tetrahydropyranyl derivatives $\mathbf{1 7}$ and $\mathbf{1 8}$ could be separated by careful flash chromatography. However, it is preferable, with these substrates, to first remove the THP group in acidic conditions $(p-\mathrm{TSA} / \mathrm{MeOH})^{[18]}$ and then to separate the corresponding allylic alcohols 19 and 20. Acylation of 19 delivered the expected allylic acetate $\mathbf{2 1}$ in $89 \%$ yield over these two steps ${ }^{[19]}$ We treated $\mathbf{2 1}$ with $\mathrm{NaH}$ followed by the palladium catalyst [prepared from $\mathrm{Pd}(\mathrm{OAc})_{2}$ and Phosphines such as monoand diphosphine $\left(\mathrm{PPh}_{3}\right.$ or dppe), phosphite or ferrocenyl Ligands]. Various solvents such as THF, toluene and DMF were tested for the present protocol. No product was detected and, in all cases, the starting material $\mathbf{2 1}$ was fully recovered. Under the same conditions, precursors bearing either a trifluoroacetate or a carbonate as leaving group also did not lead to the expected cyclohexene but degradation mostly occured.

Given the non-reactivity of the starting material, we concluded that under the operating conditions, degradation of the catalytic system should be faster than the cyclization. To test this hypothesis, we quickly heat the reaction mixture containing, the sodium anion of $\mathbf{2 1}$ and the catalyst to $90^{\circ} \mathrm{C}$. Gratifyingly, under these new reaction conditions, we obtained the desired cyclization product 22. A marked difference in reactivity was observed depending on the ligands of the palladium (Figure 2, Table 2).

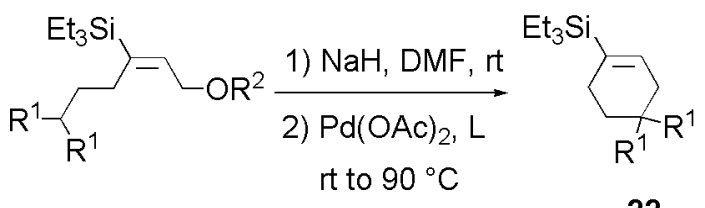

Table 2. Ligand optimization for 6-endo-trig cyclization.

\begin{tabular}{lllll}
\hline Entry & Ligand (L) & Time (h) & Conversion (\%) & ${\text { Yield }(\%)^{\mathrm{a}}}^{\text {a }}$ \\
\hline 1 & dppf & 24 & 38 & n.d. \\
2 & $\mathrm{P}(\mathrm{OPh})_{3}$ & 24 & 42 & n.d. \\
3 & dppe & 24 & 65 & n.d. \\
4 & $\mathrm{PPh}_{3}$ & 24 & 72 & 30 \\
5 & $\mathrm{P}(\mathrm{OEt})_{3}$ & 24 & 74 & 34 \\
6 & $\mathrm{P}(\mathrm{O} i \text {-Pr })_{3}$ & 2 & 100 & 66 \\
\hline
\end{tabular}

asolated yield; not determined when conversion was to low.

Regardless of the ligand used, the 6-endo-trig cyclization process has consistently held. However, the reactivities remained generally modest and the conversions did not exceed $74 \%$ after $24 \mathrm{~h}$ (Table 2, entries 1 to 5). The use of tri-isopropylphosphite allowed the full conversion of the substrate $\mathbf{2 1}$ a significant acceleration in the reaction rate as the starting material disappeared within $2 \mathrm{~h}$. It afforded the expected cyclohexene $\mathbf{2 2}$ in $66 \%$ isolated yield as well as some unidentified degradation products (Table 2, entry 6).

We also prepared a precursor having bulkier isopropyl ester functions. At this end, we performed the trans-esterification of compound 19 mediated by $\mathrm{Ti}(\mathrm{O} i \mathrm{Pr})_{4}$ in isopropanol. The expected diisopropylic malonate was isolated in $90 \%$ yield then acylated by using the standard conditions $\left(\mathrm{Ac}_{2} \mathrm{O}, \mathrm{NEt}_{3}\right)$ to provide the allylic acetate $\mathbf{2 3}$ in $96 \%$ yield (Scheme 6). Using the optimized reaction conditions (vide supra), total conversion of the starting material was observed and the corresponding cyclohexene $\mathbf{2 4}$ was isolated in a moderated $45 \%$ yield in addition to unidentified degradation products (Scheme 7).

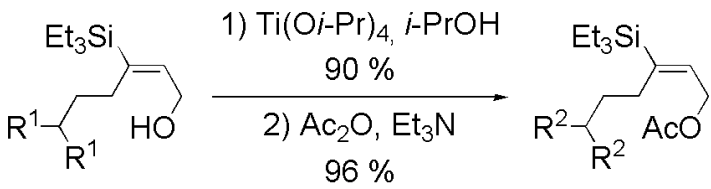

$$
\begin{aligned}
& \text { 19, } \mathrm{R}^{1}=\mathrm{CO}_{2} \mathrm{Me} \quad 23, \mathrm{R}^{2}=\mathrm{CO}_{2} \mathrm{i}-\mathrm{Pr} \\
& 23 \frac{\text { 1) } \mathrm{NaH}, \mathrm{DMF}}{\begin{array}{c}
\text { 2) } \mathrm{Pd}(\mathrm{OAc})_{2}(10 \mathrm{~mol} \%) \\
\mathrm{P}(\mathrm{Oi}-\mathrm{Pr})_{3}(40 \mathrm{~mol} \%) \\
\text { rt to } 90^{\circ} \mathrm{C}
\end{array}} \underbrace{\mathrm{R}^{2}}_{\mathrm{R}^{2}} \\
& 45 \% \\
& \text { 24, } \mathrm{R}^{2}=\mathrm{CO}_{2} \mathrm{i}-\mathrm{Pr}
\end{aligned}
$$

Scheme 7. Preparation of precursor $\mathbf{2 3}$ and cyclization into 24.

To demonstrate the strong influence of the position of the silyl group during the cyclization, we also tested the allylic acetate $\mathbf{2 5}$. This compound is supposed to produce a highly reactive $\pi$-allyl palladium complex unsuitable for cyclization. ${ }^{[1 \mathrm{a}, 12 \mathrm{~b}]}$

Thus, engaged in the palladium-catalyzed reaction at room temperature, the starting acetate $\mathbf{2 5}$ was recovered (Table 3, entry 1). As expected, upon heating, the diene $\mathbf{2 6}$ was isolated quantitatively regardless of the solvent or the ligand used (Table 3, Entries 2-6). We explain these results by the competition between the unfavorable cyclization with the fast elimination on the intermediate cationic $\pi$-allylic palladium complex. 


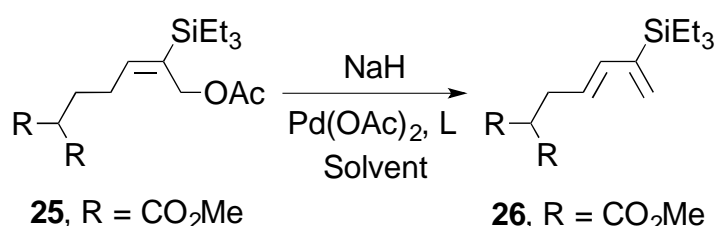

Figure 3. Palladium-catalyzed elimination into diene $\mathbf{2 6}$.

Table 3. Effect of ligand in palladium-catalyzed elimination.

\begin{tabular}{lllll}
\hline Entry & Ligand $(\mathrm{L})$ & Solvent & Temp. $\left({ }^{\circ} \mathrm{C}\right)$ & Yield $(\%)^{\mathrm{a}}$ \\
\hline 1 & $\mathrm{PPh}_{3}$ & THF & $\mathrm{rt}$ & n.r. \\
2 & $\mathrm{PPh}_{3}$ & THF & 65 & quant. \\
3 & dppe & THF & 50 & quant. \\
4 & dppe & Toluene & 80 & quant. \\
5 & dppe & DMF & 90 & quant. \\
6 & $\mathrm{P}(\mathrm{O} i \text {-Pr })_{3}$ & DMF & 90 & quant. \\
\hline
\end{tabular}

${ }^{a}$ Isolated yield.

\section{Conclusion}

We have developed conditions allowing the preparation of functionalized silylated cyclohexenes. The position of the vinylic silyl group on the starting allylic substrate strongly influenced the palladium-catalyzed cyclizations. It could favour either the production of an open chain diene by a direct elimination on the palladium intermediate or a cyclohexenyl ring via a 6-endo-trig process. In addition, the presence of a hydroxyl group at the allylic position of the starting material, allowed the cyclization to proceed smoothly. It is followed by dehydration and a 6- $\pi$ electrocyclic rearrangement giving a conjugated trienic product.

\section{Experimental Section}

The characterization data for compounds $2,3,4,5$ and 16 was previously reported. All reactions requiring anhydrous conditions were carried out under an atmosphere of nitrogen or argon in flame-dried glassware using standard syringe techniques. ${ }^{1} \mathrm{H}$ NMR spectra were recorded on 200 and $400 \mathrm{MHz}$ NMR spectrometers using $\mathrm{CDCl}_{3}(\delta=7.26 \mathrm{ppm})$ as the internal reference. ${ }^{13} \mathrm{C}$ NMR spectra were recorded at 50 or $100 \mathrm{MHz}$ using $\mathrm{CDCl}_{3}(\delta=77.16 \mathrm{ppm})$ as the internal reference. Infrared spectra were recorded on a FT-IR spectrophotometer and signals are reported in $\mathrm{cm}^{-1}$. Mass spectra were recorded either through electrospray ionization (ESI) or electron impact (EI) on an instrument operating at $70 \mathrm{eV}$.

2-Allyl-2-methoxycarbonyl-malonic acid dimethyl ester (5) ${ }^{[13]}$ At $0{ }^{\circ} \mathrm{C}$, to a suspension of $\mathrm{NaH}$ (60\% in mineral oil) $(5.85 \mathrm{~g}, 146.2 \mathrm{mmol}, 1.5$ equiv.) in THF $(150 \mathrm{~mL})$, was added $4(16.78 \mathrm{~g}, 97.4 \mathrm{mmol})$ and the mixture was warmed to r.t. and stirred during $1 \mathrm{~h}$. Then, freshly distilled $\mathrm{ClCO}_{2} \mathrm{Me}(20$ $\mathrm{mL}, 258.0 \mathrm{mmol}, 2.6$ equiv.) was slowly added at $0{ }^{\circ} \mathrm{C}$ and the solution was warmed to r.t. and stirred during $2 \mathrm{~h}$. The reaction mixture was diluted in diethyl ether and washed with saturated aqueous solution of ammonium chloride and brine, dried over $\mathrm{MgSO}_{4}$, filtered and concentrated. Pure compound 5 (20.41 g, $88.6 \mathrm{mmol}, 91 \%$ ) was obtained as a colorless oil. ${ }^{1} \mathrm{H}$ NMR $\left(\mathrm{CDCl}_{3}, 200 \mathrm{MHz}\right) \delta 5.95-5.77(\mathrm{ddt}, J=17.2 ; 10.3$ and $7.4 \mathrm{~Hz}, 1 \mathrm{H}$, $\left.\mathrm{H}_{2} \mathrm{C}=\mathrm{CHCH}_{2}\right) ; 5.09\left(\mathrm{dd}, J=17.2\right.$ and $\left.1 \mathrm{~Hz}, 1 \mathrm{H}, \mathrm{HC}=\mathrm{C} H_{\text {trans }}\right) ; 4.99(\mathrm{dd} ; J=$ 10.3 and $\left.1 \mathrm{~Hz}, 1 \mathrm{H}, \mathrm{HC}=\mathrm{CH}_{\mathrm{cis}}\right) ; 3.70\left(\mathrm{~s}, 9 \mathrm{H}, \mathrm{CO}_{2} \mathrm{CH}_{3}\right) ; 2.78(\mathrm{~d}, J=7.4 \mathrm{~Hz}$, $\left.2 \mathrm{H}, \mathrm{H}_{2} \mathrm{C}=\mathrm{CHCH}_{2}\right) .{ }^{13} \mathrm{C} \mathrm{NMR}\left(\mathrm{CDCl}_{3}, 50 \mathrm{MHz}\right) \delta 166.9\left(\mathrm{CO}_{2} \mathrm{CH}_{3}\right) ; 132.4$

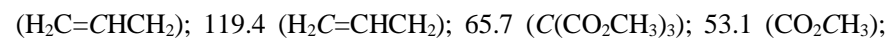
$37.6\left(\mathrm{H}_{2} \mathrm{C}=\mathrm{CHCH}_{2}\right)$. IR (neat), $\mathrm{cm}^{-1}: 2900 ; 1740 ; 1460 ; 1370 ; 1230$. Anal. for $\mathrm{C}_{10} \mathrm{H}_{14} \mathrm{O}_{6}\left(\mathrm{M}=230.22 \mathrm{~g} \cdot \mathrm{mol}^{-1}\right):$ calcd $(\%): \mathrm{C}=52.17 ; \mathrm{H}=6.13$. found (\%) : $\mathrm{C}=52.06 ; \mathrm{H}=6.32$.

2-Methoxycarbonyl-2-(2-oxo-ethyl)-malonic acid dimethyl ester (6). In a flask without septum, ozone was added to a cold $\left(-78{ }^{\circ} \mathrm{C}\right)$ solution of allyl malonate 5 (1.38 g, $6.0 \mathrm{mmol})$ in a mixture $\mathrm{CH}_{2} \mathrm{Cl}_{2} / \mathrm{MeOH}: 3 / 1(20 \mathrm{~mL})$ until a persistent blue color was perceived or until total disparition of S.M. was observed by TLC. Then, $\mathrm{O}_{3}$ in excess was removed by bubbling a $\mathrm{N}_{2}$ flow. Methyl sulfide $(2.15 \mathrm{~mL}, 30 \mathrm{mmol}, 5$ equiv.) was added and the solution is stirred $10 \mathrm{~min}$ at $-78{ }^{\circ} \mathrm{C}$, then slowly warmed to r.t. in $3 \mathrm{~h}$. $\mathrm{Me}_{2} \mathrm{~S}$ was removed under reduced pressure using a $\mathrm{NaClO}$ trap. After removal of the solvent, diethyl ether was added and the crude product which precipitate was filtered. Pure compound 6 (1.33 g, $5.7 \mathrm{mmol}, 96 \%)$ was obtained as a white powder. TLC $(\mathrm{PE} / \mathrm{EA}=7 / 3): \mathrm{R} f=0.40$. m.p. : $75{ }^{\circ} \mathrm{C} .{ }^{1} \mathrm{H} \mathrm{NMR}\left(\mathrm{CDCl}_{3}, 200\right.$ MHz) $\delta 9.68(\mathrm{~s}, 1 \mathrm{H}, \mathrm{O}=\mathrm{CH}) ; 3.78\left(\mathrm{~s}, 9 \mathrm{H}, \mathrm{CO}_{2} \mathrm{CH}_{3}\right) ; 3.17$ (s, $\left.2 \mathrm{H}, \mathrm{CH}_{2}\right) .{ }^{13} \mathrm{C}$ $\mathrm{NMR}\left(\mathrm{CDCl}_{3}, 50 \mathrm{MHz}\right) \quad \delta \square 196.5(\mathrm{O}=\mathrm{CH}) ; 166.5 \quad\left(\mathrm{CO}_{2} \mathrm{CH}_{3}\right) ; 62.4$ $\left(C\left(\mathrm{CO}_{2} \mathrm{CH}_{3}\right)_{3}\right) ; 53.9\left(\mathrm{CO}_{2} \mathrm{CH}_{3}\right) ; 45.5\left(\mathrm{CH}_{2}\right)$. IR (neat), $\mathrm{cm}^{-1}: 2975 ; 1720$; 1435; 1240. Anal. for $\mathrm{C}_{9} \mathrm{H}_{12} \mathrm{O}_{7}\left(\mathrm{M}=232.19\right.$ g.mol $\left.{ }^{-1}\right)$ : calcd $(\%): \mathrm{C}=46.56$; $\mathrm{H}=5.21$. Found $(\%): \mathrm{C}=46.68 ; \mathrm{H}=5.24$.

2-Oxo-5-[3-(tetrahydro-pyran-2-yloxy)-prop-1-ynyl]-dihydro-furan-3,3dicarboxylic acid dimethyl ester (7). To a solution of 3 (23.45 mL, 166.8 mmol, 1.1 equiv.) in THF $(250 \mathrm{~mL})$, at $-78{ }^{\circ} \mathrm{C}$, was added dropwise a $2 \mathrm{M}$ solution of $n$-BuLi ( $83.4 \mathrm{~mL}, 166.8 \mathrm{mmol}, 1.1$ equiv.) and the mixture was stirred $30 \mathrm{~min}$ at this temperature. Then, the anion was canulated onto a solution of aldehyde $6(35.2 \mathrm{~g}, 151.6 \mathrm{mmol})$ in THF $(50 \mathrm{~mL})$ and the mixture warmed to r.t. and stirred $2 \mathrm{~h}$. Then, $\mathrm{Et}_{2} \mathrm{O}$ and $\mathrm{NH}_{4} \mathrm{Cl}$ were added. The aqueous phase was extracted with $\mathrm{Et}_{2} \mathrm{O}$. Combined organic layers were washed with brine, dried over $\mathrm{MgSO}_{4}$, filtered and concentrated under reduced pressure. The crude compound $\mathbf{7}$ was obtained as an orange oil and was directly engaged in the next step. TLC $(\mathrm{PE} / \mathrm{EA}=7 / 3): \mathrm{R} f=0.35 .{ }^{1} \mathrm{H}$ $\mathrm{NMR}\left(\mathrm{CDCl}_{3}, 200 \mathrm{MHz}\right) \delta 5.31(\mathrm{t}, J=6.9 \mathrm{~Hz}, 1 \mathrm{H}, \mathrm{OCHCC}) ; 4.73(\mathrm{~s}, 1 \mathrm{H}$, $\mathrm{OCHO}$ ); 4.23 (s, $2 \mathrm{H}, \mathrm{CCCH}_{2} \mathrm{O}$ ); 3.63 (s, $\left.6 \mathrm{H}, \mathrm{CO}_{2} \mathrm{CH}_{3}\right) ; 3.60-3.42(\mathrm{~m}, 2 \mathrm{H}$, $\left.\mathrm{CH}_{2} \mathrm{CH}_{2} \mathrm{O}\right) ; 2.41\left(\mathrm{t}, J=6.9,2 \mathrm{H}, \mathrm{CH}_{2} \mathrm{CCO}_{2} \mathrm{Me}\right) ; 1.73-1.50(\mathrm{~m}, 6 \mathrm{H}$, $\left.\left(\mathrm{CH}_{2}\right)_{3} \mathrm{CHO}\right) .{ }^{13} \mathrm{C} \quad \mathrm{NMR}\left(\mathrm{CDCl}_{3}, 50 \mathrm{MHz}\right) \quad \delta \quad 168.9 \quad\left(\mathrm{CO}_{2} \mathrm{Me}\right) ; 154.5$ $\left(\mathrm{C}(\mathrm{O}) \mathrm{CCO}_{2} \mathrm{Me}\right) ; 96.8(\mathrm{OCHO}) ; 83.7\left(\mathrm{CCCH}_{2} \mathrm{OCHO}\right) ; 81.2\left(\mathrm{CCCH}_{2} \mathrm{OCHO}\right)$; 65.8 ( $\mathrm{OCHCC}) ; 62.0\left(\mathrm{CH}_{2} \mathrm{CH}_{2} \mathrm{O}\right) ; 54.0\left(\mathrm{CCCH}_{2} \mathrm{OCHO}\right) ; 52.8\left(\mathrm{CO}_{2} \mathrm{CH}_{3}\right)$; $47.8 \quad\left(\mathrm{CCO}_{2} \mathrm{Me}\right) ; \quad 33.6 \quad\left(\mathrm{CH}_{2} \mathrm{CCO}_{2} \mathrm{Me}\right) ; \quad 30.2 \quad\left(\mathrm{CH}_{2} \mathrm{CH}_{2} \mathrm{CHO}\right) ; 25.3$ $\left(\mathrm{CH}_{2} \mathrm{CH}_{2} \mathrm{CHO}\right) ; 19.0\left(\mathrm{CH}_{2} \mathrm{CH}_{2} \mathrm{O}\right)$. IR (ATR), $\mathrm{cm}^{-1}: 2953 ; 1736 ; 1439 ; 1339$; $1258 ; 1119 ; 1024 ; 941 ; 902$.

5-(3-Hydroxy-prop-1-ynyl)-2-oxo-tetrahydro-furan-3-carboxylic acid methyl ester (8). To a solution of crude $7(16.35 \mathrm{~g}, 48.03 \mathrm{mmol})$ in $\mathrm{MeOH}$ $(50 \mathrm{~mL})$ was added at r.t. $p$-TSA $(3.66 \mathrm{~g}, 19.2 \mathrm{mmol}, 40 \%)$ and the mixture was stirred overnight. Then, the solution was diluted in $\mathrm{Et}_{2} \mathrm{O}$ and a saturated aqueous solution of $\mathrm{NaHCO}_{3}$ was added. The aqueous phase was extracted with $\mathrm{Et}_{2} \mathrm{O}$. Combined organic layers were washed with brine, dried over $\mathrm{MgSO}_{4}$, filtered and concentrated under reduced pressure. The crude compound was purified by flash chromatography and $\mathbf{8}(8.92 \mathrm{~g}, 45 \mathrm{mmol} ; 93$ $\%$ over 2 steps from 6 ) was obtained as a pale yellow oil. TLC (PE/EA $=7 / 3$ ) $: \mathrm{R} f=0.10 .{ }^{1} \mathrm{H} \mathrm{NMR}\left(\mathrm{CDCl}_{3}, 400 \mathrm{MHz}\right) \delta 5.32(\mathrm{t}, J=6.4 \mathrm{~Hz}, 1 \mathrm{H}, \mathrm{OC} H \mathrm{CC})$; 4.26 (s, $\left.2 \mathrm{H}, \mathrm{CH}_{2} \mathrm{OH}\right) ; 3.77$ (s, $\left.3 \mathrm{H}, \mathrm{CO}_{2} \mathrm{CH}_{3}\right) ; 3.63$ (t, $J=7.4 \mathrm{~Hz}, 1 \mathrm{H}$, $\mathrm{CHCO}_{2} \mathrm{CH}_{3}$ ); 2.44 (dd, $J=7.4$ and $\left.6.4 \mathrm{~Hz}, 2 \mathrm{H}, \mathrm{CH}_{2} \mathrm{CHCO}_{2}\right) ; 2.23(\mathrm{~s}, 1 \mathrm{H}$, $\mathrm{OH}) .{ }^{13} \mathrm{C} \quad \mathrm{NMR} \quad\left(\mathrm{CDCl}_{3}, \quad 100 \quad \mathrm{MHz}\right) \quad \delta \quad 169.0 \quad\left(\mathrm{CO}_{2} \mathrm{Me}\right) ; \quad 154.6$ $\left(C(\mathrm{O}) \mathrm{CHCO}_{2} \mathrm{Me}\right) ; 86.1\left(\mathrm{CCCH}_{2} \mathrm{OH}\right) ; 80.4\left(\mathrm{CCCH}_{2} \mathrm{OH}\right) ; 65.8(\mathrm{OCHCC})$; $52.8\left(\mathrm{CO}_{2} \mathrm{CH}_{3}\right) ; 50.5\left(\mathrm{CH}_{2} \mathrm{OH}\right) ; 47.8\left(\mathrm{CHCO}_{2} \mathrm{Me}\right) ; 33.5\left(\mathrm{CH}_{2} \mathrm{CHCO}_{2} \mathrm{Me}\right)$. IR (neat), $\mathrm{cm}^{-1}: 3499 ; 2958 ; 1755 ; 1441 ; 1268 ; 1027 ; 939$.

2-(2,5-Dihydroxy-pent-3-ynyl)-malonic acid diisopropyl ester (9). To a solution of $8(2.47 \mathrm{~g}, 12.46 \mathrm{mmol})$ in $i$-PrOH $(20 \mathrm{~mL})$ was added, at r.t., $\mathrm{Ti}(\mathrm{O} i-\mathrm{Pr})_{4}(3.7 \mathrm{~mL}, 12.46 \mathrm{mmol}, 1$ equiv. $)$. The mixture was warmed to $80^{\circ} \mathrm{C}$ and stirred overnight. After completion of the reaction, $\mathrm{Et}_{2} \mathrm{O}$ and a saturated aqueous solution of $\mathrm{Na}_{2} \mathrm{SO}_{4}$ were added. Ti salts were filtered and the compound 9 (2.29 g, $8.0 \mathrm{mmol}, 64 \%)$ was obtained as translucent oil. TLC $(\mathrm{PE} / \mathrm{EA}=5 / 5): \mathrm{R} f=0.40 .{ }^{1} \mathrm{H} \mathrm{NMR}\left(\mathrm{CDCl}_{3}, 400 \mathrm{MHz}\right) \delta 5.26(\mathrm{t}, J=5.1 \mathrm{~Hz}$, $1 \mathrm{H}, \mathrm{CHOH}$ ); 5.05 (sept, $\left.J=6.1 \mathrm{~Hz}, 2 \mathrm{H}, \mathrm{CO}_{2} \mathrm{CH}\left(\mathrm{CH}_{3}\right)_{2}\right) ; 4.26(\mathrm{~d}, J=5.6 \mathrm{~Hz}$, $\left.2 \mathrm{H}, \mathrm{CH}_{2} \mathrm{OH}\right) ; 3.68\left(\mathrm{t}, J=8.1 \mathrm{~Hz}, 1 \mathrm{H}, \mathrm{CH}\left(\mathrm{CO}_{2} i-\mathrm{Pr}\right)_{2}\right) ; 2.88-2.46(\mathrm{~m}, 2 \mathrm{H}$, $\left.\mathrm{CH}_{2} \mathrm{CHOH}\right) ; 2.24(\mathrm{~s} \mathrm{br}, 2 \mathrm{H}, 2 \times \mathrm{CHOH}) ; 1.23(\mathrm{~d}, J=6.1 \mathrm{~Hz}, 12 \mathrm{H}$, 
$\left.\mathrm{CO}_{2} \mathrm{CH}\left(\mathrm{CH}_{3}\right)_{2}\right) .{ }^{13} \mathrm{C} \mathrm{NMR}\left(\mathrm{CDCl}_{3}, 100 \mathrm{MHz}\right) \delta 166.9\left(\mathrm{CO}_{2} \mathrm{CH}\left(\mathrm{CH}_{3}\right)_{2}\right) ; 86.8$ $(\mathrm{CCCHOH}) ; 80.9\left(\mathrm{CCCH}_{2} \mathrm{OH}\right) ; 70.4\left(\mathrm{OCH}\left(\mathrm{CH}_{3}\right)_{2}\right) ; 68.3(\mathrm{CCHOH}) ; 50.6$ $\left(\mathrm{CH}_{2} \mathrm{OH}\right) ; 46.7\left(\mathrm{CH}\left(\mathrm{CO}_{2} i-\mathrm{Pr}\right)_{2}\right) ; 33.5\left(\mathrm{CHCH}_{2}\right) ; 21.6\left(\mathrm{CO}_{2} \mathrm{CH}\left(\mathrm{CH}_{3}\right)_{2}\right)$. IR (ATR), $\mathrm{cm}^{-1}: 3482 ; 2983 ; 1778 ; 1721 ; 1454 ; 1258 ; 1147 ; 1101 ; 1007$.

General procedure A for hydrosilylation. Under $\mathrm{Ar}$, to a solution of alkyne (1 equiv.) in THF ( $3 \mathrm{M}$ ), silane (1.2 equiv.) and a $0.1 \mathrm{M}$ solution of $\mathrm{H}_{2} \mathrm{PtCl}_{6}$, $6 \mathrm{H}_{2} \mathrm{O}(0.01 \mathrm{~mol} \%)$ in THF were added. The mixture was warmed to $50{ }^{\circ} \mathrm{C}$ overnight. The solution was filtered at room temperature over a short pad of celite, concentrated in vacuo and purified by flash chromatography.

2-(2,5-Dihydroxy-3-triethylsilanyl-pent-3-enyl)-malonic acid diisopropyl ester (10) and 5-(3-Hydroxy-1-triethylsilanyl-propenyl)-2-oxo-tetrahydrofuran-3-carboxylic acid isopropyl ester (10'). These compounds were prepared according to the general procedure A from $9(1.88 \mathrm{~g}, 6.5 \mathrm{mmol})$ to give a mixture of two regioisomers $\mathbf{1 0} / \mathbf{1 1}$ in a 1/1 ratio (pale yellow oil, 2.4 $\mathrm{g}, 5.9 \mathrm{mmol}) .10$ was isolated by flash chromatography, and cyclized slowly into 10' when stored at r.t. 10: TLC $(\mathrm{PE} / \mathrm{EA}=7 / 3): \mathrm{R} f=0.40 .{ }^{1} \mathrm{H}$ NMR $\left(\mathrm{CDCl}_{3}, 400 \mathrm{MHz}\right) \delta 5.89(\mathrm{t}, J=6.1 \mathrm{~Hz}, 1 \mathrm{H},=\mathrm{CH}) ; 5.05$ (sept, $J=6.6 \mathrm{~Hz}$, $\left.2 \mathrm{H}, \mathrm{CO}_{2} \mathrm{CH}\left(\mathrm{CH}_{3}\right)_{2}\right) ; 4.53$ (dd, $J=10.6$ and $3.0 \mathrm{~Hz}, 1 \mathrm{H}, \mathrm{SiCCHOH}$ ); 4.28 (dd, $J=6.1$ and $\left.2.0 \mathrm{~Hz}, 2 \mathrm{H}, \mathrm{CH}_{2} \mathrm{OH}\right) ; 3.54\left(\mathrm{dd}, J=8.6\right.$ and $5.6 \mathrm{~Hz}, 1 \mathrm{H}, \mathrm{CHCO}_{2} i-$ Pr); $2.45(\mathrm{~s} \mathrm{br}, 1 \mathrm{H}, \mathrm{OH}) ; 2.13$ (ddd, $J=14.7,10.6$ and $5.6 \mathrm{~Hz}, 1 \mathrm{H}$, part of $\mathrm{CH}_{2} \mathrm{CHOH}$ ); 1.96 (ddd, $J=14.7,8.6$ and $3.0 \mathrm{~Hz}, 1 \mathrm{H}$, part of $\mathrm{CH}_{2} \mathrm{CHOH}$ ); 1.66 (s br, $1 \mathrm{H}, \mathrm{OH}) ; 1.25\left(\mathrm{~d}, J=6.6 \mathrm{~Hz}, 12 \mathrm{H}, \mathrm{CO}_{2} \mathrm{CH}\left(\mathrm{CH}_{3}\right)_{2}\right) ; 0.92$ (t, $J=7.6$ $\left.\mathrm{Hz}, 9 \mathrm{H}, \mathrm{SiCH}_{2} \mathrm{CH}_{3}\right) ; 0.65$ (q, $\left.J=7.6 \mathrm{~Hz}, 6 \mathrm{H}, \mathrm{SiCH}_{2} \mathrm{CH}_{3}\right) .{ }^{13} \mathrm{C} \mathrm{NMR}\left(\mathrm{CDCl}_{3}\right.$, $100 \quad \mathrm{MHz}) \quad \delta \quad 168.3 \quad\left(\mathrm{CO}_{2} i-\mathrm{Pr}\right) ; 140.6 \quad(=\mathrm{CSi}) ; 138.1 \quad(=\mathrm{CH}) ; 69.2$ $\left(\mathrm{CO}_{2} \mathrm{CH}\left(\mathrm{CH}_{3}\right)_{2}\right) ; 61.7(\mathrm{CHOH}) ; 54.8\left(\mathrm{CH}_{2} \mathrm{OH}\right) ; 49.1\left(\mathrm{CH}\left(\mathrm{CO}_{2} i-\mathrm{Pr}\right)_{2}\right) ; 33.3$ $\left(\mathrm{CH}_{2} \mathrm{CH}\left(\mathrm{CO}_{2} i-\mathrm{Pr}\right)_{2}\right) ; 21.6\left(\mathrm{CO}_{2} \mathrm{CH}\left(\mathrm{CH}_{3}\right)_{2}\right) ; 7.2\left(\mathrm{SiCH}_{2} \mathrm{CH}_{3}\right) ; 3.4\left(\mathrm{SiCH}_{2} \mathrm{CH}_{3}\right)$. IR (ATR), $\mathrm{cm}^{-1}: 3450 ; 2954 ; 2876 ; 1728 ; 1456 ; 1375 ; 1262 ; 1166 ; 1102$; 1004; 721.

10' : TLC $(\mathrm{PE} / \mathrm{EA}=7 / 3): \mathrm{R} f=0.50 .{ }^{1} \mathrm{H} \mathrm{NMR}\left(\mathrm{CDCl}_{3}, 400 \mathrm{MHz}\right) \delta 5.94(\mathrm{t}, J$ $=5.1 \mathrm{~Hz}, 1 \mathrm{H},=\mathrm{CH}) ; 5.53(\mathrm{t}, J=8.6 \mathrm{~Hz}, 1 \mathrm{H}, \mathrm{CHOCO}) ; 5.05$ (sept, $J=6.1 \mathrm{~Hz}$, $\left.1 \mathrm{H}, \mathrm{CH}\left(\mathrm{CH}_{3}\right)_{2}\right)$; 4.31-4.16 (m, 2H, $\left.\mathrm{CH}_{2} \mathrm{OH}\right) ; 3.54$ (m, $\left.1 \mathrm{H}, \mathrm{CHCO}_{2} i-\mathrm{Pr}\right) ; 2.68-$ $2.07\left(\mathrm{~m}, 2 \mathrm{H}, \mathrm{CH}_{2} \mathrm{CHO}\right) ; 1.25\left(\mathrm{~d}, J=6.1 \mathrm{~Hz}, 6 \mathrm{H}, \mathrm{CO}_{2} \mathrm{CH}\left(\mathrm{CH}_{3}\right)_{2}\right) ; 0.92(\mathrm{t}, J=$ $\left.7.6 \mathrm{~Hz}, 9 \mathrm{H}, \mathrm{SiCH}_{2} \mathrm{CH}_{3}\right) ; 0.65$ (q, $\left.J=7.6 \mathrm{~Hz}, 6 \mathrm{H}, \mathrm{SiCH}_{2} \mathrm{CH}_{3}\right) .{ }^{13} \mathrm{C}$ NMR $\left(\mathrm{CDCl}_{3}, 100 \mathrm{MHz}\right) \delta 171.8\left(\mathrm{CO}_{2} i \mathrm{-Pr}\right) ; 167.4(\mathrm{OC}=\mathrm{O}) ; 142.1(=\mathrm{CH}) ; 138.9$ $(=C \mathrm{Si}) ; 81.3(\mathrm{CHO}) ; 70.4\left(\mathrm{CO}_{2} \mathrm{CH}\left(\mathrm{CH}_{3}\right)_{2}\right) ; 59.9\left(\mathrm{CH}_{2} \mathrm{OH}\right) ; 47.7\left(\mathrm{CH}\left(\mathrm{CO}_{2} i-\right.\right.$ $\mathrm{Pr})$ ); $33.9 \quad\left(\mathrm{CH}_{2} \mathrm{CHO}\right) ; \quad 21.7 \quad\left(\mathrm{CO}_{2} \mathrm{CH}\left(\mathrm{CH}_{3}\right)_{2}\right) ; \quad 7.4 \quad\left(\mathrm{SiCH}_{2} \mathrm{CH}_{3}\right) ; \quad 3.6$ $\left(\mathrm{SiCH}_{2} \mathrm{CH}_{3}\right.$ ). IR (ATR), $\mathrm{cm}^{-1}: 2954 ; 2876 ; 1780 ; 1729 ; 1456 ; 1375 ; 1256$; 1164; 1103; 1004; 971; 722. Anal. for $\mathrm{C}_{17} \mathrm{H}_{30} \mathrm{O}_{5} \mathrm{Si}\left(\mathrm{M}=342.50\right.$ g.mol $\left.{ }^{-1}\right)$ : calcd $(\%): \mathrm{C}=59.62 ; \mathrm{H}=8.83$. found $(\%): \mathrm{C}=59.71 ; \mathrm{H}=8.74$

General procedure B : 2-(5-Acetoxy-2-hydroxy-3-triethylsilanyl-pent-3enyl)-malonic acid diisopropyl ester (12). To a solution of $\mathbf{1 0}(430 \mathrm{mg}, 1.15$ mmol), in dry $\mathrm{CH}_{2} \mathrm{Cl}_{2}(2 \mathrm{~mL})$, distilled $\mathrm{Et}_{3} \mathrm{~N}(0.4 \mathrm{~mL}, 2.8 \mathrm{mmol}, 2.5$ equiv. $)$ was added and the resulting suspension was allowed to stir at room temperature until dissolution was completed. Freshly distilled acetyl chloride (80 $\square \mathrm{L}, 1.15 \mathrm{mmol}, 1$ equiv.) was then added dropwise at $0^{\circ} \mathrm{C}$ to the reaction mixture. The reaction was then allowed to warm to room temperature and stirred overnight. The mixture was treated with a saturated aqueous solution of $\mathrm{NH}_{4} \mathrm{Cl}$. The aqueous phase was extracted with $\mathrm{CH}_{2} \mathrm{Cl}_{2}$. The collected organic layers were washed with brine, dried over $\mathrm{MgSO}_{4}$ and evaporated in vacuo. The crude product was purified by flash chromatography to give $\mathbf{1 2}$ $(511 \mathrm{mg}, 1.15 \mathrm{mmol}$, quant) as a colorless oil. TLC $($ Pent/EA = 95/5) $: \mathrm{R} f=$ $0.60 .{ }^{1} \mathrm{H} \mathrm{NMR}\left(\mathrm{CDCl}_{3}, 400 \mathrm{MHz}\right) \delta 5.81(\mathrm{t}, J=6.8 \mathrm{~Hz}, 1 \mathrm{H},=\mathrm{CH}) ; 5.54(\mathrm{~m}$,

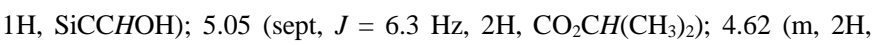
$\mathrm{CH}_{2} \mathrm{OAc}$ ); 3.51 (m, $1 \mathrm{H}, \mathrm{CHCO}_{2} i-\mathrm{Pr}$ ); 2.69-2.37 (m, 2H, $\left.\mathrm{CH}_{2} \mathrm{CHOH}\right) ; 2.05$ (s, $\left.3 \mathrm{H}, \mathrm{OCOCH}_{3}\right) ; 1.27$ (d, $\left.J=6.3 \mathrm{~Hz}, 12 \mathrm{H}, \mathrm{CO}_{2} \mathrm{CH}\left(\mathrm{CH}_{3}\right)_{2}\right) ; 0.90$ (t, $J=7.8 \mathrm{~Hz}$, $\left.9 \mathrm{H}, \mathrm{SiCH}_{2} \mathrm{CH}_{3}\right) ; 0.66$ (q, $\left.J=7.8 \mathrm{~Hz}, 6 \mathrm{H}, \mathrm{SiCH}_{2} \mathrm{CH}_{3}\right) .{ }^{13} \mathrm{C} \mathrm{NMR}_{\left(\mathrm{CDCl}_{3}, 100\right.}$ $\mathrm{MHz}) \delta 171.6\left(\mathrm{OCOCH}_{3}\right) ; 167.3\left(\mathrm{CO}_{2} i-\mathrm{Pr}\right) ; 141.7(=C \mathrm{Si}) ; 136.3(=\mathrm{CH}) ; 81.2$ $(\mathrm{CHO}) ; 70.5 \quad\left(\mathrm{CO}_{2} \mathrm{CH}\left(\mathrm{CH}_{3}\right)_{2}\right) ; 61.3\left(\mathrm{CH}_{2} \mathrm{O}\right) ; 47.7 \quad\left(\mathrm{CH}\left(\mathrm{CO}_{2} i-\mathrm{Pr}\right) ; 33.7\right.$ $\left(\mathrm{CH}_{2} \mathrm{CHO}\right) ; 21.8\left(\mathrm{CO}_{2} \mathrm{CH}\left(\mathrm{CH}_{3}\right)_{2}\right) ; 21.1\left(\mathrm{OCOCH}_{3}\right) ; 7.5\left(\mathrm{SiCH}_{2} \mathrm{CH}_{3}\right) ; 3.6$ $\left(\mathrm{SiCH}_{2} \mathrm{CH}_{3}\right.$ ). IR (ATR), $\mathrm{cm}^{-1}: 3440 ; 2954 ; 2873 ; 1727 ; 1455 ; 1375 ; 1260$; 1168; 1101; 1004; 720. Anal. for $\mathrm{C}_{22} \mathrm{H}_{40} \mathrm{O}_{7} \mathrm{Si}\left(\mathrm{M}=444.63 \mathrm{~g} . \mathrm{mol}^{-1}\right)$ : calcd (\%) $: \mathrm{C}=59.43 ; \mathrm{H}=9.07$. found $(\%): \mathrm{C}=59.69 ; \mathrm{H}=8.68$.

5-(3-Acetoxy-1-triethylsilanyl-propenyl)-2-oxo-tetrahydro-furan-3carboxylic acid isopropyl ester (12'). The formation of this compound (pale yellow oil) sometime occurs during acylation of $\mathbf{1 0}$ or when $\mathbf{1 2}$ was stored at r.t. $\mathrm{TLC}(\mathrm{PE} / \mathrm{EA}=7 / 3): \mathrm{R} f=0.30 .{ }^{1} \mathrm{H} \mathrm{NMR}\left(\mathrm{CDCl}_{3}, 400 \mathrm{MHz}\right) \delta 5.83(\mathrm{t}, J$ $=5.4 \mathrm{~Hz}, 1 \mathrm{H},=\mathrm{CH}) ; 5.54(\mathrm{t}, J=6.6 \mathrm{~Hz}, 1 \mathrm{H}, \mathrm{CHOCO}) ; 5.05($ sept,$J=6.1 \mathrm{~Hz}$, $\left.1 \mathrm{H}, \mathrm{CH}\left(\mathrm{CH}_{3}\right)_{2}\right) ; 4.65-4.58\left(\mathrm{~m}, 2 \mathrm{H}, \mathrm{CH}_{2} \mathrm{O}\right) ; 3.57$ (m, $\left.1 \mathrm{H}, \mathrm{CHCO}_{2} i-\mathrm{Pr}\right) ; 2.70-$ $2.37\left(\mathrm{~m}, 2 \mathrm{H}, \mathrm{CH}_{2} \mathrm{CHO}\right) ; 2.07\left(\mathrm{~s}, 3 \mathrm{H}, \mathrm{OCOCH}_{3}\right) ; 1.24(\mathrm{~d}, J=6.1 \mathrm{~Hz}, 6 \mathrm{H}$, $\left.\mathrm{CO}_{2} \mathrm{CH}\left(\mathrm{CH}_{3}\right)_{2}\right) ; 0.90$ (t, $\left.J=7.6 \mathrm{~Hz}, 9 \mathrm{H}, \mathrm{SiCH}_{2} \mathrm{CH}_{3}\right) ; 0.62(\mathrm{q}, J=7.6 \mathrm{~Hz}, 6 \mathrm{H}$, $\left.\mathrm{SiCH}_{2} \mathrm{CH}_{3}\right) .{ }^{13} \mathrm{C}$ NMR $\left(\mathrm{CDCl}_{3}, 100 \mathrm{MHz}\right) \delta 172.5(\mathrm{OCOCH}) ; 171.6$ $\left(\mathrm{OCOCH}_{3}\right) ; 167.8\left(\mathrm{CO}_{2} i-\mathrm{Pr}\right) ; 142.4(=\mathrm{CSi}) ; 137.1(=\mathrm{CH}) ; 81.9(\mathrm{CHO}) ; 71.2$ $\left(\mathrm{CO}_{2} \mathrm{CH}\left(\mathrm{CH}_{3}\right)_{2}\right) ; 62.0\left(\mathrm{CH}_{2} \mathrm{O}\right) ; 48.4\left(\mathrm{CH}\left(\mathrm{CO}_{2} i-\mathrm{Pr}\right) ; 34.5\left(\mathrm{CH}_{2} \mathrm{CHO}\right) ; 22.6\right.$ $\left(\mathrm{CO}_{2} \mathrm{CH}\left(\mathrm{CH}_{3}\right)_{2}\right) ; 21.8\left(\mathrm{OCOCH}_{3}\right) ; 8.2\left(\mathrm{SiCH}_{2} \mathrm{CH}_{3}\right) ; 4.4\left(\mathrm{SiCH}_{2} \mathrm{CH}_{3}\right)$. IR (ATR), $\mathrm{cm}^{-1}: 2954 ; 2876 ; 1782 ; 1733 ; 1455 ; 1375 ; 1227 ; 1163 ; 1105 ; 1019$; 970; 722. Anal. for $\mathrm{C}_{19} \mathrm{H}_{32} \mathrm{O}_{6} \mathrm{Si}\left(\mathrm{M}=384.54 \mathrm{~g} \cdot \mathrm{mol}^{-1}\right)$ : $\operatorname{calcd}(\%): \mathrm{C}=59.34 ; \mathrm{H}$ $=8.39$. found $(\%): \mathrm{C}=59.27 ; \mathrm{H}=8.40$.

General procedure C: 2-(3-Triethylsilanyl-penta-2,4-dienylidene)malonic acid diisopropyl ester (15). To a suspension of sodium hydride (19 $\mathrm{mg}, 0.475 \mathrm{mmol}, 0.95$ equiv. $)$ in $\mathrm{THF}(2 \mathrm{~mL})$, was added, at $0{ }^{\circ} \mathrm{C}$, a solution of $12(215 \mathrm{mg}, 0.5 \mathrm{mmol})$ in THF $(0.5 \mathrm{~mL})$. The mixture was warmed to r.t. and stirred during $10 \mathrm{~min}$. Then, a beforehand made solution of $\mathrm{Pd}(\mathrm{OAc})_{2}(6$ $\mathrm{mg}, 5 \mathrm{~mol} \%), \mathrm{PPh}_{3}(26 \mathrm{mg}, 20 \mathrm{~mol} \%)$ in THF $(2 \mathrm{~mL}$ ) was added and the mixture was warmed to reflux. After $1 \mathrm{~h}, \mathrm{Et}_{2} \mathrm{O}$ and $\mathrm{NH}_{4} \mathrm{Cl}$ were added and after a standard work-up, the crude product was purified by flash chromatography and $\mathbf{1 5}(77 \mathrm{mg}, 0.21 \mathrm{mmol}, 42 \%)$ was obtained as a colorless oil. TLC (pent./AE $=95 / 5): \mathrm{R} f=0.95 .{ }^{1} \mathrm{H} \mathrm{NMR}\left(\mathrm{CDCl}_{3}, 400 \mathrm{MHz}\right) \delta 7.74$ $\left(\mathrm{d}, J=12.2 \mathrm{~Hz}, 1 \mathrm{H}, \mathrm{CHCCO}_{2} i-\mathrm{Pr}\right) ; 6.68(\mathrm{dd}, J=17.3$ et $11.2 \mathrm{~Hz}, 1 \mathrm{H}$, $\left.\mathrm{CHCH}_{2}\right) ; 6.63(\mathrm{~d}, J=12.2 \mathrm{~Hz}, 1 \mathrm{H}, \mathrm{SiC}=\mathrm{C} H) ; 5.34(\mathrm{~d}, J=11.2 \mathrm{~Hz}, 1 \mathrm{H}$, $\left.\mathrm{CH}=\mathrm{CH}_{\text {cis }}\right) ; 5.22\left(\mathrm{~d}, J=17.3 \mathrm{~Hz}, 1 \mathrm{H}, \mathrm{CH}=\mathrm{CH}_{\text {trans }}\right) ; 5.20$ and $5.09(2 \mathrm{sept}, J=$ $\left.6.1 \mathrm{~Hz}, 2 \times 1 \mathrm{H}, 2 \times \mathrm{CO}_{2} \mathrm{CH}\left(\mathrm{CH}_{3}\right)_{2}\right) ; 1.31$ and $1.25(2 \mathrm{~d}, J=6.1 \mathrm{~Hz}, 2 \times 6 \mathrm{H}$, $\left.2 \times \mathrm{CO}_{2} \mathrm{CH}\left(\mathrm{CH}_{3}\right)_{2}\right) ; 0.90\left(\mathrm{t}, J=8.1 \mathrm{~Hz}, 9 \mathrm{H}, \mathrm{CH}_{2} \mathrm{CH}_{3}\right) ; 0.69(\mathrm{q}, J=8.1 \mathrm{~Hz}, 6 \mathrm{H}$,

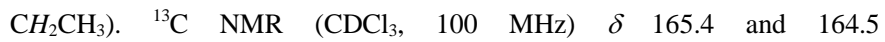
$\left(2 \times \mathrm{CO}_{2} \mathrm{CH}\left(\mathrm{CH}_{3}\right)_{2}\right) ; 154.4\left(\mathrm{CCO}_{2} i-\mathrm{Pr}\right) ; 137.6\left(\mathrm{CHCCO}_{2} i-\mathrm{Pr}\right) ; 135.7\left(\mathrm{CHCH}_{2}\right)$; $132.9(\mathrm{SiC}=\mathrm{CH}) ; 127.2\left(\mathrm{CH}=\mathrm{CH}_{2}\right) ; 119.8 \quad(\mathrm{SiC}=\mathrm{CH}) ; 69.1$ and 68.9 $\left(2 \times \mathrm{CO}_{2} \mathrm{CH}\left(\mathrm{CH}_{3}\right)_{2}\right) ; 21.9\left(2 \times \mathrm{CO}_{2} \mathrm{CH}\left(\mathrm{CH}_{3}\right)_{2}\right) ; 7.4\left(\mathrm{CH}_{2} \mathrm{CH}_{3}\right) ; 3.3\left(\mathrm{CH}_{2} \mathrm{CH}_{3}\right)$. IR (neat), $\mathrm{cm}^{-1}: 2950 ; 1720 ; 1605 ; 1375 ; 1255 ; 1110 ; 740$. HRMS (IC, $\mathrm{CH}_{4}$ ) for $\left[\mathrm{MNH}_{4}\right]^{+}$367.2305, mes. 367.2307.

2-[5-(Tetrahydro-pyran-2-yloxy)-3-triethylsilanyl-pent-3-enyl]-malonic acid dimethyl ester (17) and 2-[5-(Tetrahydro-pyran-2-yloxy)-4-triethylsilanylpent-3-enyl]-malonic acid dimethyl ester (18). These compounds were prepared according to the general procedure A from 2-[5-(tetrahydro-pyran-2yloxy)-pent-3-ynyl]-malonic acid dimethyl ester $\mathbf{1 6}^{[21]}$ (13.13 g, $\left.44 \mathrm{mmol}\right)$. After completion of the reaction, a 2/3 mixture of two regioisomers $\mathbf{1 7}$ and $\mathbf{1 8}$ was obtained $(16.18 \mathrm{~g}, 39 \mathrm{mmol}) .17: \mathrm{TLC}(\mathrm{PE} / \mathrm{EA}=7 / 3): \mathrm{R} f=0.70 .{ }^{1} \mathrm{H}$ $\mathrm{NMR}\left(\mathrm{CDCl}_{3}, 400 \mathrm{MHz}\right) \delta 5.83(\mathrm{t}, J=5.6 \mathrm{~Hz}, 1 \mathrm{H},=\mathrm{CH}) ; 4.53(\mathrm{~m}, 1 \mathrm{H}$, $\mathrm{OCHO}$ ); 4.26 (dd, part of $\mathrm{AB}$ system, $J=13.0$ and $5.6 \mathrm{~Hz}, 1 \mathrm{H},=\mathrm{CHCH}_{2} \mathrm{O}$ ); $4.06\left(\mathrm{dd}\right.$, part of $\mathrm{AB}$ system, $J=13.0$ and $\left.6.6 \mathrm{~Hz}, 1 \mathrm{H},=\mathrm{CHCH}_{2} \mathrm{O}\right) ; 3.68(\mathrm{~s}$, $\left.6 \mathrm{H}, \mathrm{CO}_{2} \mathrm{CH}_{3}\right) ; 3.45\left(\mathrm{~m}, 2 \mathrm{H}, \mathrm{CH}_{2} \mathrm{CH}_{2} \mathrm{O}\right) ; 3.30\left(\mathrm{t}, \mathrm{J}=7.6 \mathrm{~Hz}, 1 \mathrm{H}, \mathrm{CHCO}_{2} \mathrm{CH}_{3}\right)$; $2.12\left(\mathrm{~m}, 2 \mathrm{H}, \mathrm{CH}_{2} \mathrm{CH}_{2} \mathrm{CSi}\right) ; 1.93\left(\mathrm{~m}, 2 \mathrm{H}, \mathrm{CH}_{2} \mathrm{CSi}\right) ; 1.9-1.4(\mathrm{~m}, 6 \mathrm{H}$, $\mathrm{CH}_{2} \mathrm{CH}_{2} \mathrm{CH}_{2} \mathrm{CH}$ ); 0.91 (t, $\left.J=7.6 \mathrm{~Hz}, 9 \mathrm{H}, \mathrm{CH}_{2} \mathrm{CH}_{3}\right) ; 0.56$ (q, $J=7.6 \mathrm{~Hz}, 6 \mathrm{H}$, $\left.\mathrm{CH}_{2} \mathrm{CH}_{3}\right) .{ }^{13} \mathrm{C} \mathrm{NMR}\left(\mathrm{CDCl}_{3}, 100 \mathrm{MHz}\right) \delta 169.6\left(\mathrm{CO}_{2} \mathrm{CH}_{3}\right) ; 141.4$ (=CSi); $139.3(=\mathrm{CH}) ; 98.1 \quad(\mathrm{OCHO}) ; 66.1 \quad\left(=\mathrm{CHCH}_{2} \mathrm{O}\right) ; 62.4 \quad\left(\mathrm{CH}_{2} \mathrm{CH}_{2} \mathrm{O}\right) ; 52.5$ $\left(\mathrm{CO}_{2} \mathrm{CH}_{3}\right) ; 51.8\left(\mathrm{CHCO}_{2} \mathrm{Me}\right) ; 41.1\left(\mathrm{OCHCH}_{2}\right) ; 30.7\left(\mathrm{CH}_{2} \mathrm{CH}_{2} \mathrm{OCHO}\right) ; 28.7$ $\left(\mathrm{CH}_{2} \mathrm{CHCO}_{2} \mathrm{Me}\right) ; 25.6 \quad\left(\mathrm{CH}_{2} \mathrm{CH}_{2} \mathrm{CHCO}_{2} \mathrm{Me}\right) ; 19.6 \quad\left(\mathrm{CH}_{2} \mathrm{CH}_{2} \mathrm{CHO}\right) ; 6.6$ $\left(\mathrm{SiCH}_{2} \mathrm{CH}_{3}\right) ; 2.9\left(\mathrm{SiCH}_{2} \mathrm{CH}_{3}\right)$. IR (ATR), $\mathrm{cm}^{-1}: 2953 ; 2923 ; 1739 ; 1436$; 1228; 1151; 1070; 1003; 733. 18: TLC (PE/EA $=7 / 3): \mathrm{R} f=0.70 .{ }^{1} \mathrm{H}$ NMR $\left(\mathrm{CDCl}_{3}, 400 \mathrm{MHz}\right) \delta 5.83(\mathrm{t}, J=5.6 \mathrm{~Hz}, 1 \mathrm{H},=\mathrm{CH}) ; 4.53(\mathrm{~m}, 1 \mathrm{H}, \mathrm{OCHO})$; $4.30\left(\mathrm{~d}, 1 \mathrm{H}, J=11.7 \mathrm{~Hz}, 1 \mathrm{H}\right.$, part of $\left.\mathrm{CH}_{2} \mathrm{O}\right) ; 3.92(\mathrm{~d}, J=11.7 \mathrm{~Hz}, 1 \mathrm{H}$, part of $\left.\mathrm{CH}_{2} \mathrm{O}\right) ; 3.68\left(\mathrm{~s}, 6 \mathrm{H}, \mathrm{CO}_{2} \mathrm{CH}_{3}\right) ; 3.45\left(\mathrm{~m}, 2 \mathrm{H}, \mathrm{CH}_{2} \mathrm{CH}_{2} \mathrm{O}\right) ; 3.30(\mathrm{t}, J=7.6 \mathrm{~Hz}$, $\left.1 \mathrm{H}, \mathrm{CHCO}_{2} \mathrm{CH}_{3}\right) ; 2.13\left(\mathrm{~m}, 2 \mathrm{H},=\mathrm{CHCH}_{2} \mathrm{CH}_{2}\right) ; 1.95\left(\mathrm{~m}, 2 \mathrm{H},=\mathrm{CHCH}_{2}\right) ; 1.9-$ 1.4 (m, 6H, $\mathrm{CH}_{2} \mathrm{CH}_{2} \mathrm{CH}_{2} \mathrm{CH}$ ); 0.83 (t, $\left.J=7.8 \mathrm{~Hz}, 9 \mathrm{H}, \mathrm{CH}_{2} \mathrm{CH}_{3}\right) ; 0.55$ (q, $J=$ $\left.7.8 \mathrm{~Hz}, 6 \mathrm{H}, \mathrm{CH}_{2} \mathrm{CH}_{3}\right) .{ }^{13} \mathrm{C} \mathrm{NMR}\left(\mathrm{CDCl}_{3}, 100 \mathrm{MHz}\right) \delta 169.8\left(\mathrm{CO}_{2} \mathrm{CH}_{3}\right) ; 139.6$ $(=C \mathrm{Si}) ; 137.1(=\mathrm{CH}) ; 98.1(\mathrm{OCHO}) ; 62.4\left(\mathrm{CH}_{2} \mathrm{CH}_{2} \mathrm{O}\right) ; 61.7\left(\mathrm{SiCCH}_{2} \mathrm{O}\right) ; 52.5$ $\left(\mathrm{CO}_{2} \mathrm{CH}_{3}\right) ; 51.1\left(\mathrm{CHCO}_{2} \mathrm{Me}\right) ; 41.1\left(\mathrm{OCHCH}_{2}\right) ; 30.7\left(\mathrm{CH}_{2} \mathrm{CH}_{2} \mathrm{OCHO}\right) ; 30.6$ $\left(\mathrm{CH}_{2} \mathrm{CH}_{2} \mathrm{CHCO}_{2} \mathrm{Me}\right) ; 26.8\left(\mathrm{CH}_{2} \mathrm{CHCO}_{2} \mathrm{Me}\right) ; 19.3 \quad\left(\mathrm{CH}_{2} \mathrm{CH}_{2} \mathrm{CHO}\right) ; 7.5$ $\left(\mathrm{SiCH}_{2} \mathrm{CH}_{3}\right) ; 3.3\left(\mathrm{SiCH}_{2} \mathrm{CH}_{3}\right)$.

General procedure D: 2-(5-Hydroxy-pent-3-ynyl)-malonic acid dimethyl ester $^{[18]}$ (19). To a solution of $\mathbf{1 6}(1.49 \mathrm{~g}, 5.0 \mathrm{mmol})$ in $\mathrm{MeOH}(10 \mathrm{~mL})$ was added at r.t. $p$-TSA $(95 \mathrm{mg}, 0.5 \mathrm{mmol}, 10 \mathrm{~mol} \%)$ and the mixture was stirred 
overnight. Then, the solution was diluted in $\mathrm{Et}_{2} \mathrm{O}$ and a saturated aqueous solution of $\mathrm{NaHCO}_{3}$ was added. The aqueous phase was extracted with $\mathrm{Et}_{2} \mathrm{O}$. Combined organic layers were washed with brine, dried over $\mathrm{MgSO}_{4}$, filtered and concentrated under reduced pressure. The crude compound was purified by flash chromatography to give $19(1.07 \mathrm{~g}, 5.0 \mathrm{mmol}$, quant.) as a pale yellow oil. NMR spectrum were identical to the described molecule TLC $(\mathrm{PE} / \mathrm{EA}=7 / 3): \mathrm{R} f=0.20 .{ }^{1} \mathrm{H} \mathrm{NMR}\left(\mathrm{CDCl}_{3}, 400 \mathrm{MHz}\right) \delta 4.18(\mathrm{~s}, 2 \mathrm{H}$, $\mathrm{CH}_{2} \mathrm{OH}$ ); 3.70 (s, $\left.6 \mathrm{H}, \mathrm{CO}_{2} \mathrm{CH}_{3}\right) ; 3.54$ (t, $\left.J=7.1 \mathrm{~Hz}, 1 \mathrm{H}, \mathrm{CHCO}_{2} \mathrm{CH}_{3}\right) ; 2.42$ (s, $1 \mathrm{H}, \mathrm{OH}) ; 2.27\left(\mathrm{t}, J=7.1 \mathrm{~Hz}, 2 \mathrm{H}, \mathrm{CCCH}_{2} \mathrm{CH}_{2}\right) ; 2.05$ (dt, $J=7.1$ and 7.1 $\left.\mathrm{Hz}, 2 \mathrm{H}, \mathrm{CH}_{2} \mathrm{CHCO}_{2} \mathrm{Me}\right) .{ }^{13} \mathrm{C} \mathrm{NMR}\left(\mathrm{CDCl}_{3}, 100 \mathrm{MHz}\right) \delta 169.6\left(\mathrm{CO}_{2} \mathrm{CH}_{3}\right)$; $83.9\left(\mathrm{CCCH}_{2} \mathrm{OH}\right) ; 80.1\left(\mathrm{CCCH}_{2} \mathrm{OH}\right) ; 52.7\left(\mathrm{CO}_{2} \mathrm{CH}_{3}\right) ; 51.1\left(\mathrm{CH}_{2} \mathrm{OH}\right) ; 50.4$ $\left(\mathrm{CHCO}_{2} \mathrm{CH}_{3}\right) ; 27.6\left(\mathrm{CCCH}_{2} \mathrm{CH}_{2}\right) ; 16.8\left(\mathrm{CCCH}_{2} \mathrm{CH}_{2}\right)$. IR (ATR), $\mathrm{cm}^{-1}$ : 3419; $2955 ; 1728 ; 1435 ; 1248 ; 1154 ; 1047 ; 1010$.

2-(5-Hydroxy-3-triethylsilanyl-pent-3-enyl)-malonic acid dimethylester (19) and 2-(5-Hydroxy-4-triethylsilanyl-pent-3-enyl)-malonic acid dimethyl ester (20). Following general procedure D, a 2 / 3 mixture of 17 and $18(53.9 \mathrm{~g}, 130 \mathrm{mmol})$ delivered two regioisomers 19 (16.8 g, 50.8 $\mathrm{mmol})$ and 20 (26.0 g, $78.7 \mathrm{mmol})$ which were separated on silicagel.

19: $\mathrm{TLC}(\mathrm{PE} / \mathrm{EA}=6 / 4): \mathrm{R} f=0.65 .{ }^{1} \mathrm{H} \mathrm{NMR}\left(\mathrm{CDCl}_{3}, 200 \mathrm{MHz}\right) \delta 5.89(\mathrm{t}, J=$ $6.1 \mathrm{~Hz}, 1 \mathrm{H},=\mathrm{CH}) ; 4.21\left(\mathrm{~d}, J=6.1 \mathrm{~Hz}, 2 \mathrm{H}, \mathrm{CH}_{2} \mathrm{OH}\right) ; 3.70\left(\mathrm{~s}, 6 \mathrm{H}, \mathrm{CO}_{2} \mathrm{CH}_{3}\right)$; $3.31\left(\mathrm{t}, J=7.6 \mathrm{~Hz}, 1 \mathrm{H}, \mathrm{CH}\left(\mathrm{CO}_{2} \mathrm{CH}_{3}\right)_{2}\right) ; 2.05\left(\mathrm{~m}, 2 \mathrm{H}, \mathrm{CH}_{2} \mathrm{CH}_{2} \mathrm{CSi}\right) ; 2.02$ (s, $1 \mathrm{H}, \mathrm{OH}) ; 1.81\left(\mathrm{~m}, 2 \mathrm{H}, \mathrm{CH}_{2} \mathrm{CSi}\right) ; 0.87\left(\mathrm{t}, J=8.1 \mathrm{~Hz}, 9 \mathrm{H}, \mathrm{CH}_{2} \mathrm{CH}_{3}\right) ; 0.55$ (q, $J$ $\left.=8.1 \mathrm{~Hz}, 6 \mathrm{H}, \mathrm{CH}_{2} \mathrm{CH}_{3}\right) .{ }^{13} \mathrm{C} \mathrm{NMR}\left(\mathrm{CDCl}_{3}, 50 \mathrm{MHz}\right) \delta 169.7\left(\mathrm{CO}_{2} \mathrm{CH}_{3}\right)$; $141.8 \quad(=\mathrm{CH}) ; \quad 139.1 \quad(=\mathrm{CSi}) ; \quad 59.3 \quad\left(\mathrm{CH}_{2} \mathrm{OH}\right) ; \quad 52.6 \quad\left(\mathrm{CO}_{2} \mathrm{CH}_{3}\right) ; \quad 51.6$ $\left(\mathrm{CH}\left(\mathrm{CO}_{2} \mathrm{CH}_{3}\right)_{2}\right) ; 29.1\left(\mathrm{CH}_{2} \mathrm{CH}_{2} \mathrm{CSi}\right) ; 27.9\left(\mathrm{CH}_{2} \mathrm{CSi}\right) ; 7.4\left(\mathrm{CH}_{2} \mathrm{CH}_{3}\right) ; 2.9$ $\left(\mathrm{CH}_{2} \mathrm{CH}_{3}\right)$. IR (neat), $\mathrm{cm}^{-1}: 3446 ; 2954 ; 2911 ; 2874 ; 1736 ; 1437 ; 1268 ; 1158$; 1005; 737. Anal. for $\mathrm{C}_{16} \mathrm{H}_{30} \mathrm{O}_{5} \mathrm{Si}\left(\mathrm{M}=330.49 \mathrm{~g} \cdot \mathrm{mol}^{-1}\right)$ : calcd $(\%): \mathrm{C}=58.15$; $\mathrm{H}=9.15$. found $(\%): \mathrm{C}=58.01 ; \mathrm{H}=9.16$.

20: $\mathrm{TLC}(\mathrm{PE} / \mathrm{EA}=6 / 4): \mathrm{R} f=0.85 .{ }^{1} \mathrm{H} \mathrm{NMR}\left(\mathrm{CDCl}_{3}, 200 \mathrm{MHz}\right) \delta 5.73(\mathrm{t}, J=$ $7.1 \mathrm{~Hz}, 1 \mathrm{H},=\mathrm{CH}) ; 4.15\left(\mathrm{~s}, 2 \mathrm{H}, \mathrm{CH}_{2} \mathrm{OH}\right) ; 3.71\left(\mathrm{~s}, 6 \mathrm{H}, \mathrm{CO}_{2} \mathrm{CH}_{3}\right) ; 3.34(\mathrm{t}, J=$ $\left.7.6 \mathrm{~Hz}, 1 \mathrm{H}, \mathrm{CH}\left(\mathrm{CO}_{2} \mathrm{CH}_{3}\right)_{2}\right) ; 2.21\left(\mathrm{dt}, J=7.6\right.$ and $\left.7.1 \mathrm{~Hz}, 2 \mathrm{H}, \mathrm{CH}_{2} \mathrm{CH}_{2} \mathrm{CH}=\right)$; $1.97\left(\mathrm{dt}, J=7.6\right.$ and $\left.7.1 \mathrm{~Hz}, 2 \mathrm{H}, \mathrm{CH}_{2} \mathrm{CH}_{2} \mathrm{CH}=\right) ; 1.53$ (s, $1 \mathrm{H}, \mathrm{OH}$ ); 0.89 (t, $J=$

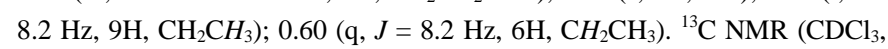
$50 \mathrm{MHz}) \delta 169.9\left(\mathrm{CO}_{2} \mathrm{CH}_{3}\right) ; 142.1(=\mathrm{CH}) ; 139.9(=\mathrm{CSi}) ; 60.4\left(\mathrm{CH}_{2} \mathrm{OH}\right) ; 52.7$ $\left(\mathrm{CO}_{2} \mathrm{CH}_{3}\right) ; 51.0\left(\mathrm{CH}\left(\mathrm{CO}_{2} \mathrm{CH}_{3}\right)_{2}\right) ; 28.5\left(\mathrm{CH}_{2} \mathrm{CH}_{2} \mathrm{CH}=\right) ; 26.3\left(\mathrm{CH}_{2} \mathrm{CH}_{2} \mathrm{CH}=\right)$; $7.5\left(\mathrm{CH}_{2} \mathrm{CH}_{3}\right) ; 3.2\left(\mathrm{CH}_{2} \mathrm{CH}_{3}\right)$. IR (neat), $\mathrm{cm}^{-1}$ : 3474; 2953; 2877; 2361; 1733; 1437; 1229 (br); 1156; 1005; 716. Anal. for $\mathrm{C}_{16} \mathrm{H}_{30} \mathrm{O}_{5} \mathrm{Si}\left(\mathrm{M}=330.49\right.$ g.mol $\left.{ }^{-1}\right)$ : calcd $(\%): \mathrm{C}=58.15 ; \mathrm{H}=9.15$. found $(\%): \mathrm{C}=58.10 ; \mathrm{H}=9.10$.

2-(5-Acetoxy-3-triethylsilanyl-pent-3-enyl)-malonic acid dimethyl ester (21).

Method A: Following general procedure B, from allylic alcohol 19 (350 mg, $1.06 \mathrm{mmol}$ ). The product 21 (395 $\mathrm{mg}, 1.06 \mathrm{mmol}$ ) was quantitatively obtained as an colorless oil.

Method $B:{ }^{[19]}$ Acetic anhydride $(0.45 \mathrm{~mL}, 4.8 \mathrm{mmol}, 1.2$ equiv.) was added dropwise to a solution of pyranyl protected compound $\mathbf{1 7}(1.66 \mathrm{~g}, 4 \mathrm{mmol})$ and $\mathrm{Cu}(\mathrm{OTf})_{2}(72.3 \mathrm{mg}, 0.2 \mathrm{mmol}, 5 \mathrm{~mol} \%)$ in $\mathrm{CH}_{2} \mathrm{Cl}_{2}(20 \mathrm{~mL})$ and was stirred at r.t. overnight. The reaction mixture was diluted with $\mathrm{CH}_{2} \mathrm{Cl}_{2}$ and washed with sodium bicarbonate and brine. The organic layer was dried over anhydrous sodium sulfate and concentrated on a rotary evaporator. The crude product was purified over silica gel by flash chromatography to provide the pure acetate 21 ( $863 \mathrm{mg}, 2.32 \mathrm{mmol}, 58 \%)$ as a pale yellow oil. TLC ( = PE / EA) : $\mathrm{R} f=0.95 .{ }^{1} \mathrm{H} \mathrm{NMR}\left(\mathrm{CDCl}_{3}, 400 \mathrm{MHz}\right) \delta 5.80(\mathrm{t}, J=6.1 \mathrm{~Hz}, 1 \mathrm{H},=\mathrm{CH})$; $4.63\left(\mathrm{~d}, J=6.1 \mathrm{~Hz}, 2 \mathrm{H}, \mathrm{CH}_{2} \mathrm{O}\right) ; 3.71\left(\mathrm{~s}, 6 \mathrm{H}, \mathrm{CO}_{2} \mathrm{CH}_{3}\right) ; 3.33(\mathrm{t}, J=7.6 \mathrm{~Hz}$, $\left.1 \mathrm{H}, \mathrm{CH}\left(\mathrm{CO}_{2} \mathrm{CH}_{3}\right)_{2}\right) ; 2.11\left(\mathrm{~m}, 2 \mathrm{H}, \mathrm{CH}_{2} \mathrm{CSi}\right) ; 2.03\left(\mathrm{~s}, 3 \mathrm{H}, \mathrm{COCH}_{3}\right) ; 1.83(\mathrm{~m}$, $2 \mathrm{H}, \mathrm{CH}_{2} \mathrm{CH}_{2} \mathrm{CSi}$ ); 0.91 (t, $\left.J=7.6 \mathrm{~Hz}, 9 \mathrm{H}, \mathrm{CH}_{2} \mathrm{CH}_{3}\right) ; 0.60(\mathrm{q}, J=7.6 \mathrm{~Hz}, 6 \mathrm{H}$, $\left.\mathrm{CH}_{2} \mathrm{CH}_{3}\right) .{ }^{13} \mathrm{C} \mathrm{NMR}\left(\mathrm{CDCl}_{3}, 100 \mathrm{MHz}\right) \delta 171.0\left(\mathrm{COCH}_{3}\right) ; 169.6\left(\mathrm{CO}_{2} \mathrm{CH}_{3}\right)$; $142.0 \quad(=\mathrm{CH}) ; \quad 136.0 \quad(=\mathrm{CSi}) ; \quad 61.0 \quad\left(\mathrm{CH}_{2} \mathrm{O}\right) ; \quad 52.6 \quad\left(\mathrm{CO}_{2} \mathrm{CH}_{3}\right) ; \quad 51.6$ $\left(\mathrm{CH}\left(\mathrm{CO}_{2} \mathrm{CH}_{3}\right)_{2}\right) ; 29.0\left(\mathrm{CH}_{2} \mathrm{CH}_{2} \mathrm{CSi}\right) ; 28.0\left(\mathrm{CH}_{2} \mathrm{CSi}\right) ; 21.1\left(\mathrm{COCH}_{3}\right) ; 7.4$ $\left(\mathrm{CH}_{2} \mathrm{CH}_{3}\right) ; 2.8\left(\mathrm{CH}_{2} \mathrm{CH}_{3}\right)$. IR (ATR), $\mathrm{cm}^{-1}: 2954 ; 2875 ; 1735 ; 1435 ; 1225$; $1154 ; 1017 ; 717$.

4-Triethylsilanyl-cyclohex-3-ene-1,1-dicarboxylic acid dimethyl ester (22). To a solution of $\mathrm{NaH}(80 \mathrm{mg}, 2 \mathrm{mmol}, 1$ equiv., $60 \%$ in mineral oil) in freshly distilled and degassed DMF $(0.5 \mathrm{~mL})$, was added, at $0{ }^{\circ} \mathrm{C}$, a solution of allylic acetate $21(745 \mathrm{mg}, 2 \mathrm{mmol})$ in DMF $(0.5 \mathrm{~mL})$. The mixture was warmed to r.t. and stirred $15 \mathrm{~min}$. In a second flask, $\mathrm{Pd}(\mathrm{OAc})_{2}(44.9 \mathrm{mg}, 0.2$ mmol, $10 \mathrm{~mol} \%)$ was dissolved in DMF $(1 \mathrm{~mL})$. Distilled $\mathrm{P}(\mathrm{O} i-\mathrm{Pr})_{3}(0.2$ $\mathrm{mL}, 0.8 \mathrm{mmol}, 40 \mathrm{~mol} \%$ ) was added dropwise. After the first drop of phosphite, the mixture become dark but at the end of the addition it took on a pale yellow colour. The catalyst was stirred $5 \mathrm{~min}$ and added onto the first mixture. The mixture was immediately warmed to $90{ }^{\circ} \mathrm{C}$ (in a beforehand warmed oil bath). The completion of the reaction was followed by TLC (revealed by $\mathrm{KMnO}_{4}$ ). After $2 \mathrm{~h}$, the mixture was filtered over celite and silica then the DMF was evaporated under reduced pressure $\left(30^{\circ} \mathrm{C}\right.$ under 0.5 $\mathrm{mmHg}$ ) and the crude product was purified by flash chromatography to give $22(413 \mathrm{mg}, 1.32 \mathrm{mmol})$ as a colorless oil. TLC $(\mathrm{PE} / \mathrm{EA}=9 / 1): \mathrm{R} f=0.90 .{ }^{1} \mathrm{H}$ NMR $\left(\mathrm{CDCl}_{3}, 400 \mathrm{MHz}\right) \delta 5.89(\mathrm{tt}, J=3.6$ and $2.0 \mathrm{~Hz}, 1 \mathrm{H},=\mathrm{CH}) ; 3.69(\mathrm{~s}$, $\left.6 \mathrm{H}, \mathrm{CO}_{2} \mathrm{CH}_{3}\right) ; 2.58\left(\mathrm{~d}, \mathrm{~J}=3.6 \mathrm{~Hz}, 2 \mathrm{H},=\mathrm{CHCH}_{2}\right) ; 2.09\left(\mathrm{~m}, 2 \mathrm{H}, \mathrm{CH}_{2} \mathrm{CH}_{2} \mathrm{CSi}\right.$ ); $2.07\left(\mathrm{~m}, 2 \mathrm{H}, \mathrm{CH}_{2} \mathrm{CSi}\right.$ ); $0.86\left(\mathrm{t}, J=7.6 \mathrm{~Hz}, 9 \mathrm{H}, \mathrm{CH}_{2} \mathrm{CH}_{3}\right) ; 0.52$ (q, $J=7.6 \mathrm{~Hz}$, $\left.6 \mathrm{H}, \mathrm{CH}_{2} \mathrm{CH}_{3}\right) .{ }^{13} \mathrm{C} \mathrm{NMR}\left(\mathrm{CDCl}_{3}, 100 \mathrm{MHz}\right) \delta 172.3\left(\mathrm{CO}_{2} \mathrm{CH}_{3}\right) ; 135.0$ (=CSi); $133.4(=\mathrm{CH}) ; 53.1 \quad\left(\mathrm{CCO}_{2} \mathrm{CH}_{3}\right) ; 52.6\left(\mathrm{CO}_{2} \mathrm{CH}_{3}\right) ; 32.2\left(=\mathrm{CHCH}_{2}\right) ; 28.1$ $\left(=\mathrm{CSiCH}_{2} \mathrm{CH}_{2}\right) ; 24.8\left(=\mathrm{CSiCH}_{2}\right) ; 7.4\left(\mathrm{CH}_{2} \mathrm{CH}_{3}\right) ; 2.5\left(\mathrm{CH}_{2} \mathrm{CH}_{3}\right)$. IR (neat), $\mathrm{cm}^{-1}: 2952 ; 2874 ; 1736 ; 1619 ; 1238 ; 716$. Anal. for $\mathrm{C}_{16} \mathrm{H}_{28} \mathrm{O}_{4} \mathrm{Si}(\mathrm{M}=312.48$ g. $\left.\mathrm{mol}^{-1}\right): \operatorname{calcd}(\%): \mathrm{C}=61.50 ; \mathrm{H}=9.03$. found $(\%): \mathrm{C}=61.32 ; \mathrm{H}=9.11$.

2-(5-Acetoxy-3-triethylsilanyl-pent-3-enyl)-malonic acid diisopropyl ester (23). To a solution of 19 (330.5 mg, $1 \mathrm{mmol})$ in $i-\mathrm{PrOH}(3 \mathrm{~mL})$, was added distilled $\mathrm{Ti}(\mathrm{O} i \text {-Pr })_{4}(0.3 \mathrm{~mL}, 1 \mathrm{mmol}, 1$ equiv. $)$ and the mixture was warmed to $80{ }^{\circ} \mathrm{C}$ overnight. After completion of the reaction, $\mathrm{Et}_{2} \mathrm{O}$ and a saturated aqueous solution of $\mathrm{Na}_{2} \mathrm{SO}_{4}$ were added. Ti salts were filtered and the crude allylic alcohol (351 mg, $0.9 \mathrm{mmol})$ was obtained as a translucent oil pure enough for direct conversion into the corresponding acetate. To a solution of allylic alcohol (187 mg, $0.48 \mathrm{mmol})$, in dry $\mathrm{CH}_{2} \mathrm{Cl}_{2}(2.5 \mathrm{~mL})$, distilled $\mathrm{Et}_{3} \mathrm{~N}$ ( $0.25 \mathrm{~mL}, 1.78 \mathrm{mmol}, 3.7$ equiv.) was added and the resulting suspension was allowed to stir at room temperature until dissolution was completed. Acetic anhydride ( $70 \square \mathrm{L}, 0.75 \mathrm{mmol}, 1.5$ equiv.) was added dropwise at $0^{\circ} \mathrm{C}$ and the reaction mixture was allowed to warm to room temperature and stirred overnight. The mixture was treated with a saturated aqueous solution of $\mathrm{NH}_{4} \mathrm{Cl}$. The aqueous phase was extracted with $\mathrm{CH}_{2} \mathrm{Cl}_{2}$. The collected organic layers were washed with brine, dried over $\mathrm{MgSO}_{4}$ and evaporated in vacuo. The crude product was purified by flash chromatography to give $\mathbf{2 3}$ (199 mg, $0.48 \mathrm{mmol}, 96 \%)$ as a colorless oil. TLC $(\mathrm{PE} / \mathrm{EA}=7 / 3): \mathrm{R} f=0.95 .{ }^{1} \mathrm{H}$ $\mathrm{NMR}\left(\mathrm{CDCl}_{3}, 400 \mathrm{MHz}\right) \delta 5.80(\mathrm{t}, J=6.1 \mathrm{~Hz}, 1 \mathrm{H},=\mathrm{CH}) ; 5.05$ (sept, $J=6.2$ $\left.\mathrm{Hz}, 2 \times 1 \mathrm{H}, \mathrm{CO}_{2} \mathrm{CH}\left(\mathrm{CH}_{3}\right)_{2}\right) ; 4.67\left(\mathrm{~d}, J=6.1 \mathrm{~Hz}, 2 \mathrm{H}, \mathrm{CH}_{2} \mathrm{O}\right) ; 3.22(\mathrm{t}, J=7.6$ $\left.\mathrm{Hz}, 1 \mathrm{H}, \mathrm{CH}\left(\mathrm{CO}_{2} i-\mathrm{Pr}\right)_{2}\right) ; 2.13\left(\mathrm{~m}, 2 \mathrm{H}, \mathrm{CH}_{2} \mathrm{CSi}\right) ; 2.05$ (s, $\left.3 \mathrm{H}, \mathrm{COCH}_{3}\right) ; 1.82$ (m, $\left.2 \mathrm{H}, \mathrm{CH}_{2} \mathrm{CH}_{2} \mathrm{CSi}\right) ; 1.23\left(\mathrm{~d}, J=6.2 \mathrm{~Hz}, 2 \times 6 \mathrm{H}, \mathrm{CO}_{2} \mathrm{CH}\left(\mathrm{CH}_{3}\right)_{2}\right) ; 0.89(\mathrm{t}, J=$

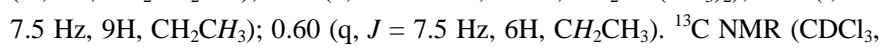
$100 \mathrm{MHz}) \delta 171.0\left(\mathrm{COCH}_{3}\right) ; 168.9\left(\mathrm{CO}_{2} i-\mathrm{Pr}\right) ; 142.2(=C \mathrm{Si}) ; 136.0(=\mathrm{CH})$; $69.0\left(\mathrm{CO}_{2} \mathrm{CH}\left(\mathrm{CH}_{3}\right)_{2}\right) ; 61.2\left(\mathrm{CH}_{2} \mathrm{O}\right) ; 52.5\left(\mathrm{CH}\left(\mathrm{CO}_{2} i-\mathrm{Pr}\right)_{2}\right) ; 28.8\left(\mathrm{CH}_{2} \mathrm{CH}_{2} \mathrm{CSi}\right)$; $28.1\left(\mathrm{CH}_{2} \mathrm{CSi}\right) ; 21.8\left(\mathrm{CO}_{2} \mathrm{CH}\left(\mathrm{CH}_{3}\right)_{2}\right) ; 21.2\left(\mathrm{COCH}_{3}\right) ; 7.5\left(\mathrm{CH}_{2} \mathrm{CH}_{3}\right) ; 2.9$ $\left(\mathrm{CH}_{2} \mathrm{CH}_{3}\right)$. IR (neat), $\mathrm{cm}^{-1}: 2953 ; 2876 ; 1727 ; 1466 ; 1374 ; 1227 ; 1102 ; 717$. Anal. for $\mathrm{C}_{22} \mathrm{H}_{40} \mathrm{O}_{6} \mathrm{Si}\left(\mathrm{M}=428.63\right.$ g.mol $\left.{ }^{-1}\right)$ : calcd $(\%): \mathrm{C}=61.65 ; \mathrm{H}=9.41$. found $(\%): \mathrm{C}=61.67 ; \mathrm{H}=9.49$.

4-Triethylsilanyl-cyclohex-3-ene-1,1-dicarboxylic acid diisopropyl ester (24). This compound was prepared following the general procedure C. From 23 (215 mg, $0.5 \mathrm{mmol})$, pure compound 24 (84 mg, $0.23 \mathrm{mmol}, 45 \%)$ was obtained as a colorless oil TLC $(\mathrm{PE} / \mathrm{EA}=9 / 1): \mathrm{R} f=0.90 .{ }^{1} \mathrm{H} \mathrm{NMR}\left(\mathrm{CDCl}_{3}\right.$, $400 \mathrm{MHz}) \delta 5.90(\mathrm{t}, J=3.6 \mathrm{~Hz}, 1 \mathrm{H},=\mathrm{CH}$ ); 4.99 (sept, $J=6.1 \mathrm{~Hz}, 2 \times 1 \mathrm{H}$, $\left.\mathrm{CO}_{2} \mathrm{CH}\left(\mathrm{CH}_{3}\right)_{2}\right) ; 2.54\left(\mathrm{~d}, J=3.6 \mathrm{~Hz}, 2 \mathrm{H},=\mathrm{CHCH}_{2}\right) ; 2.05(\mathrm{~s}, 2 \times 2 \mathrm{H}$, $\left.=\mathrm{CSiCH}_{2} \mathrm{CH}_{2}\right) ; 1.19\left(2 \times \mathrm{d}, J=6.1 \mathrm{~Hz}, 12 \mathrm{H}, \mathrm{CO}_{2} \mathrm{CH}\left(\mathrm{CH}_{3}\right)_{2}\right) ; 0.86(\mathrm{t}, J=7.6$ $\left.\mathrm{Hz}, 9 \mathrm{H}, \mathrm{SiCH}_{2} \mathrm{CH}_{3}\right) ; 0.51$ (q, $\left.J=7.6 \mathrm{~Hz}, 6 \mathrm{H}, \mathrm{SiCH}_{2} \mathrm{CH}_{3}\right) .{ }^{13} \mathrm{C} \mathrm{NMR}\left(\mathrm{CDCl}_{3}\right.$, $100 \mathrm{MHz}) \delta 171.4\left(\mathrm{CO}_{2} \mathrm{CH}\left(\mathrm{CH}_{3}\right)_{2}\right) ; 134.8(=\mathrm{CSi}) ; 133.7(=\mathrm{CH}) ; 68.6$ $\left(\mathrm{CO}_{2} \mathrm{CH}\left(\mathrm{CH}_{3}\right)_{2}\right) ; 53.0\left(\mathrm{C}\left(\mathrm{CO}_{2} i-\mathrm{Pr}\right)_{2}\right) ; 32.0\left(=\mathrm{CHCH}_{2}\right) ; 27.8\left(\mathrm{SiCCH}_{2} \mathrm{CH}_{2}\right)$; $24.7\left(\mathrm{SiCCH}_{2}\right) ; 21.7\left(\mathrm{CO}_{2} \mathrm{CH}\left(\mathrm{CH}_{3}\right)_{2}\right) ; 7.5\left(\mathrm{CH}_{2} \mathrm{CH}_{3}\right) ; 2.5\left(\mathrm{CH}_{2} \mathrm{CH}_{3}\right)$. IR (neat), $\mathrm{cm}^{-1}: 2952 ; 2875 ; 1727 ; 1619 ; 1466 ; 1285 ; 1172 ; 1145 ; 715$. Anal. for $\mathrm{C}_{20} \mathrm{H}_{36} \mathrm{O}_{4} \mathrm{Si}\left(\mathrm{M}=368.58\right.$ g.mol $\left.{ }^{-1}\right): \operatorname{calcd}(\%): \mathrm{C}=65.17 ; \mathrm{H}=9.84$. found $(\%)$ $: \mathrm{C}=65.21 ; \mathrm{H}=9.69$.

2-(5-Acetoxy-4-triethylsilanyl-pent-3-enyl)-malonic acid dimethyl ester (25). This compound was prepared quantitatively following the general procedure B, from $20(330 \mathrm{mg}, 1 \mathrm{mmol})$. TLC $(\mathrm{PE} / \mathrm{EA}=7 / 3): \mathrm{R} f=0.95 .{ }^{1} \mathrm{H}$ 
NMR $\left(\mathrm{CDCl}_{3}, 400 \mathrm{MHz}\right) \delta 5.87(\mathrm{t}, J=7.1 \mathrm{~Hz}, 1 \mathrm{H},=\mathrm{CH}) ; 4.67(\mathrm{~s}, 2 \mathrm{H}$ $\left.\mathrm{CH}_{2} \mathrm{O}\right) ; 3.76\left(\mathrm{~s}, 6 \mathrm{H}, \mathrm{CO}_{2} \mathrm{CH}_{3}\right) ; 3.39$ (t, $\left.J=7.6 \mathrm{~Hz}, 1 \mathrm{H}, \mathrm{CH}\left(\mathrm{CO}_{2} \mathrm{CH}_{3}\right)_{2}\right) ; 2.23$ (dd, $J=15.3$ and $\left.7.1 \mathrm{~Hz}, 2 \mathrm{H},=\mathrm{CHCH}_{2}\right) ; 2.06\left(\mathrm{~s}, 3 \mathrm{H}, \mathrm{COCH}_{3}\right) ; 2.02(\mathrm{dd}, J=$ 15.3 and $7.6 \mathrm{~Hz}, 2 \mathrm{H}, \mathrm{CH}_{2} \mathrm{CH}_{2} \mathrm{CH}=$ ); 0.93 (t, $J=7.6 \mathrm{~Hz}, 9 \mathrm{H}, \mathrm{CH}_{2} \mathrm{CH}_{3}$ ); 0.62 $\left(\mathrm{q}, J=7.6 \mathrm{~Hz}, 6 \mathrm{H}, \mathrm{CH}_{2} \mathrm{CH}_{3}\right),{ }^{13} \mathrm{C} \mathrm{NMR}\left(\mathrm{CDCl}_{3}, 100 \mathrm{MHz}\right) \delta 170.9$ : $\left(\mathrm{OCOCH}_{3}\right)$; 169.7: $\left(\mathrm{CO}_{2} \mathrm{CH}_{3}\right)$; 143.8: $(=C \mathrm{H}) ; 134.4:(=C \mathrm{Si}) ; 62.9:\left(\mathrm{CH}_{2} \mathrm{O}\right)$; 52.6: $\left(\mathrm{CO}_{2} \mathrm{CH}_{3}\right) ; \quad$ 51.0: $\quad\left(\mathrm{CH}\left(\mathrm{CO}_{2} \mathrm{CH}_{3}\right)_{2}\right) ; \quad 28.5: \quad\left(\mathrm{CH}_{2} \mathrm{CH}_{2} \mathrm{CH}=\right) ; \quad 26.7$ : $\left(\mathrm{CH}_{2} \mathrm{CH}=\right)$; 21.0: $\left(\mathrm{COCH}_{3}\right) ; 7.3:\left(\mathrm{CH}_{2} \mathrm{CH}_{3}\right) ; 3.1:\left(\mathrm{CH}_{2} \mathrm{CH}_{3}\right)$. IR (neat), $\mathrm{cm}^{-1}$ : $3459 ; 2954 ; 2911 ; 2875 ; 1739 ; 1436 ; 1231 ; 1156 ; 1021 ; 736$. Anal. for $\mathrm{C}_{18} \mathrm{H}_{32} \mathrm{O}_{6} \mathrm{Si}\left(\mathrm{M}=372.53 \mathrm{~g} \cdot \mathrm{mol}^{-1}\right):$ calcd $(\%): \mathrm{C}=58.03 ; \mathrm{H}=8.66$. found $(\%)$ :C $=57.98 ; \mathrm{H}=8.77$.

2-(4-Triethylsilanyl-penta-2,4-dienyl)-malonic acid dimethyl ester (26). The formation of this compound occurs when compound 25 was submitted to the palladium-catalyzed general procedure C. TLC $(\mathrm{PE} / \mathrm{EA}=7 / 3): \mathrm{R} f=0.80$. ${ }^{1} \mathrm{H} \mathrm{NMR}\left(\mathrm{CDCl}_{3}, 400 \mathrm{MHz}\right) \delta 6.18(\mathrm{~d}, J=15.2 \mathrm{~Hz}, 1 \mathrm{H}, \mathrm{CH}=\mathrm{CHCSi}) ; 5.70$ (d, $\left.J=3.1 \mathrm{~Hz}, 1 \mathrm{H}, \mathrm{SiC}=\mathrm{C} H_{\text {trans }}\right) ; 5.59(\mathrm{dt}, J=15.2$ and $7.1 \mathrm{~Hz}, 1 \mathrm{H}$, $\left.\mathrm{CH}=\mathrm{CHC}=\mathrm{CH}_{2}\right) ; 5.29\left(\mathrm{~d}, J=3.1 \mathrm{~Hz}, 1 \mathrm{H}, \mathrm{SiC}=\mathrm{CH}_{\mathrm{cis}}\right) ; 3.69\left(\mathrm{~s}, 6 \mathrm{H}, \mathrm{CO}_{2} \mathrm{CH}_{3}\right)$; $3.42\left(\mathrm{t}, J=7.6 \mathrm{~Hz}, 1 \mathrm{H}, \mathrm{CHCO}_{2} \mathrm{Me}\right.$ ); 2.63 (dd, $J=7.6$ and $7.1 \mathrm{~Hz}, 2 \mathrm{H}$, $\mathrm{CH}_{2} \mathrm{CHCO}_{2} \mathrm{Me}$ ); 0.88 (t, $\left.J=7.6 \mathrm{~Hz}, 9 \mathrm{H}, \mathrm{SiCH}_{2} \mathrm{CH}_{3}\right) ; 0.61$ (q, $J=7.6 \mathrm{~Hz}, 6 \mathrm{H}$, $\left.\mathrm{SiCH}_{2} \mathrm{CH}_{3}\right) .{ }^{13} \mathrm{C} \mathrm{NMR}\left(\mathrm{CDCl}_{3}, 100 \mathrm{MHz}\right) \delta 169.3\left(\mathrm{CO}_{2} \mathrm{CH}_{3}\right) ; 145.5$ (=CSi); $138.4(\mathrm{CH}=C \mathrm{HCSi}) ; 128.8\left(\mathrm{SiC}=\mathrm{CH}_{2}\right) ; 125.9(\mathrm{CH}=\mathrm{CHCSi}) ; 52.5\left(\mathrm{CO}_{2} \mathrm{CH}_{3}\right)$; $51.9\left(\mathrm{CHCO}_{2} \mathrm{Me}\right) ; 32.5\left(\mathrm{CH}_{2} \mathrm{CHCO}_{2} \mathrm{Me}\right) ; 7.3\left(\mathrm{SiCH}_{2} \mathrm{CH}_{3}\right) ; 2.0\left(\mathrm{SiCH}_{2} \mathrm{CH}_{3}\right)$. IR (ATR), $\mathrm{cm}^{-1}: 2952 ; 2924 ; 2874 ; 1737 ; 1435 ; 1228 ; 1151 ; 1003 ; 967$.

\section{Acknowledgments}

We thank the University P. et M. Curie and the European Commission for funding of this research through a PhD grant to C.C. and a EMEA Erasmus Mundus Scholarship to J.A. The authors would like to thank Drs B. Malezieux and L. Dechoux for fruitful discussions.

\section{References and notes}

1. Books on palladium-catalyzed reactions: (a) Tsuji, J. Palladium Reagents and Catalysis: Innovations in Organic Synthesis, Wiley: Chichester, U.K., 1995. (b) Tsuji, J. Perspectives in Organopalladium Chemistry for the 21st Century, Elsevier: Amsterdam, 1999. (c) Tsuji, J. Palladium Reagents and Catalysis, New Perspectives for the 21st Century, Wiley, Chichester, U.K., 2004. (d) Negishi, E. Handbook of Organopalladium Chemistry for Organic Synthesis, John Wiley \& Sons: New York, 2002.

2. (a) Tsuji, J.; Takahashi, H.; Morikawa, M. Tetrahedron Lett. 1965, 4387-4388. (b) Trost, B. M.; Fullerton, T. J. J. Am. Chem. Soc. 1973, 95, 292-294. d) Trost, B. M.; Dietsch, T. J. J. Am. Chem. Soc. 1973, 95, 8200-8201.

3. (a) Trost, B. M.; Crawley, M. L. Chem. Rev. 2003, 103, 29212943. (b) Weaver, J. D.; Recio, A.; Grenning, A. J.; Tunge, J. A. Chem. Rev. 2011, 111, 1846-1913. (c) Magano, J.; Dunetz, J. R. Chem. Rev. 2011, 111, 2177-2250 (d) Trost, B. M. Org. Process. Res. Dev. 2012, 16, 185-194. (e) Lumbroso, A.; Cooke, M. L.; Breit, B. Angew. Chem. Int. Ed. 2013, 52, 1890-1932. (f) Bandini, M.; Cera, G.; Chiarucci, M. Synthesis 2012, 504-512; (g) see also: Ohmatsu, K.; Ito, M.; Kunieda, T.; Ooi, T. J. Am. Chem. Soc. 2013, 135, 590-593.

4. (a) Prat, M.; Ribas, J.; Moreno-Mañas, M. Tetrahedron 1992, 48, 1695-1706. (b) Moreno-Mañas, M.; Pajuelo, F.; Parella, T.; Pleixats, R. Organometallics 1997, 16, 205-209. (c) Branchadell, V.; Moreno-Mañas, M.; Pajuelo, F.; Pleixats, R. Organometallics 1999, 18, 4934-4941. (d) Delbecq, F.; Lepouge, C. Organometallics 2000, 19, 2716-2723. (e) Norsikian, S.; Chang, C.-W. Current Org. Synth. 2009, 6, 264-289.

5. (a) see ref 1d, Chapter V. (b) Trost, B. M.; Verhoeven, T. R. J. Am. Chem. Soc. 1977, 99, 3867-3868. (c) Ahmar, M.; Cazes, B.; Goré, J. Tetrahedron 1987, 43, 3453-3463. (d) Trost, B. M. Angew. Chem. Int. Engl. Ed. 1989, 28, 1173-1192. (e) Tenaglia, A.; Kammerer, F. Synlett 1996, 576-578. (f) Transition metal catalysed enantioselective allylic substitution in organic synthesis.; In Topic Organomet. Chem.; Kazmaier U., Ed.; Springer-Verlag Berlin Heidelberg Publisher, 2012, Vol. 38, 345p. (g) Inuki, S Total Synthesis of Bioactive Natural Product By Palladium-Catalyzed Domino Cyclization of Allenes and Related Compounds. Springer Theses 2012. (h) Majumdar, K. C.;
Chattopadhyay, B.; Maji, P. K.; Chattopadhyay, S. K.; Samanta, S Heterocycles 2010, 81, 795-866.

6. (a) Baldwin, J. E. J. Chem. Soc., Chem. Commun. 1976, 734-736. (b) Baldwin, J. E.; Cutting, J.; Dupont, W.; Kruse, L.; Silberman, L.; Thomas, R. C. J. Chem. Soc., Chem. Commun. 1976, 736-738. (c) Baldwin, J. E. J. Chem. Soc., Chem. Commun. 1976, 738-741. (d) see also a recent discussion: Alabugin, I. V.; Gilmore, K.; Manoharan, M. J. Am. Chem. Soc. 2011, 133, 12608-12623. (e) Daly, M.; Cant, A. A.; Fowler, L. S.; Simpson, G. L.; Hans, M.; Sutherland, A. J. Org. Chem. 2012, 77, 10001-10009. (f) Yao, H.; Ren, J.; Tong, R. Chem. Commun. 2013, 49, 193-195.

7. Zucco, M.; Le Bideau, F.; Malacria, M. Tetrahedron Lett. 1995, 36, 2487-2490.

8. (a) Monfette, S.; Fogg, D. E. Chem. Rev. 2009, 109, 3783-3816. (b) Prunet, J. Eur. J. Org. Chem. 2011, 20, 3634-3647. (c) Peeck, L. H.; Savka, R. D.; Plenio, H. Chem. Eur. J. 2012, 18, 1284512853.

9. (a) Hirao, T.; Enda, J.; Ohshiro, Y.; Agawa, T. Tetrahedron Lett. 1981, 22, 3079-3080. (b) Trost, B. M.; Self, C. R. J. Am. Chem. Soc. 1983, 105, 5942-5944. (c) Trost, B. M.; Brandi, A. J. Org. Chem. 1984, 49, 4811-4816. (d) Otha, T.; Hosokawa, T.; Murahashi, S.-I.; Miki, K.; Kasai, N. Organometallics 1985, 4, 2080-2085. (e) Tsuji, J.; Yuhara, M.; Minato, M.; Yamada, H.; Sato, F.; Kobayashi, Y. Tetrahedron Lett. 1988, 29, 343-346. (f) Inami, H.; Ito, T.; Urabe, H.; Sato, F. Tetrahedron lett. 1993, 34, 5919-5922. (g) Schobert, R.; Barnickel, B. Synthesis 2009, 27782784. For theoretical approaches see : (h) Branchadell, V.; Moreno-Mañas, M.; Pleixats, R. Organometallics 2002, 21, 24072412. For a review on the interaction between $\beta$-substituents (including silicon groups) and the allyl moiety of palladium complexes, see: (i) Szabó, K. J. Chem. Soc. Rev. 2001, 30, 136143.

10. (a) Thorimbert, S.; Malacria, M. Tetrahedron Lett. 1996, 37, 8483-8480. (b) Commandeur, C.; Thorimbert, S.; Malacria, M. J. Org. Chem. 2003, 68, 5588-5592. (c) Branchadell, V.; MorenoMañas, M.; Pleixats, R.; Thorimbert, S.; Commandeur, C.; Boglio, C.; Malacria, M. J. Organomet. Chem. 2003, 687, 337-345.

11. (a) Thorimbert, S.; Taillier, C.; Bareyt, S.; Humilière, D.; Malacria, M. Tetrahedron Lett. 2004, 45, 9123-9126. (b) Boglio, C.; Stahlke, S.; Thorimbert, S.; Malacria, M. Org. Lett. 2005, 7, 4851-4854. (c) Lamas, M.-C.; Malacria, M.; Thorimbert, S. Eur. J. Org. Chem. 2011, 2777-2780.

12. (a) Thorimbert, S.; Malacria, M. Tetrahedron Lett. 1998, 39, 9659-9660. (b) Poli, G.; Giambastiani, G.; Malacria, M.; Thorimbert, S. Tetrahedron Lett. 2001, 42, 6287-6289. (c) Thorimbert, S.; Giambastiani, G.; Commandeur, C.; Vitale, M.; Poli, G.; Malacria, M. Eur. J. Org. Chem. 2003, 2702-2708.

13. (a) Boehme, H.; Haefner, L. Chem. Ber. 1966, 99, 879-884. b) Brillon, D. Synthetic Commun. 1986, 16, 291-298. c) Cravotto, G.; Giovenzana, G.B.; Sisti, M.; Palmisano, G. Tetrahedron 1998, 54, 1639-1646.

14. (a) Speier, J. L.; Webster, J. A.; Barnes, G. H. J. Am. Chem. Soc. 1957, 79, 974-979. For some recent studies see : (b) Li, J.; Suh , J. M.; Chin, E. Org. Lett. 2010, 12, 4712-4715. (c) Kawasaki, Y.; Ishikawa, Y.; Igawa, K.; Tomooka, K. J. Am. Chem. Soc. 2011, 133, 20712-20715 (d) Rooke, D. A.; Ferreira, E. M. Angew. Chem. Int. Ed. 2012, 51, 3225-3230.

15. Control reactions in the absence of palladium confirmed the nonreactivity of the starting allylic precursors without catalyst.

16. (a) Kinoshita, H.; Shinokubo, H.; Oshima, K. Org. Lett. 2004, 6, 4085-4088. (b) Usui, I.; Schmidt, S.; Keller, M.; Breit, B. Org. Lett. 2008, 10, 1207-1210. (c) Gosh, R.; Sarkar, A. J. Org. Chem. 2011, 76, 8508-8512.

17. For $8-\pi$ electrocyclization with vinyl silanes see (a) Salem, B. Suffert, J. Angew. Chem. Int. Ed. 2004, 43, 2826-2830. (b) Bour, C.; Blond, G.; Salem, B.; Suffert, J. Tetrahedron, 2006, 62, 10567-10581. See also two recent reviews dealing with electrocyclizations (c) Beaudry, C. M.; Malerich, J. P.; Trauner, D. Chem. Rev. 2005, 105, 4757-4778. (d) Thompson, S.; Coyne, A. C.; Knipe, P. C.; Smith, M. D. Chem. Soc. Rev. 2011, 40, 4217-4231.

18. (a) Brillon, D.; Deslongchamps, P. Can. J. Chem. 1987, 65, 4345. (b) Brillon, D.; Deslongchamps, P. Can. J. Chem. 1987, 65, 56-68. see also : (c) Burns, C. J.; Gill, M.; Saubern, S. Aust. J. Chem. 1997, 50, 1067-1080 (d) Mukai, C.; Kuroda, N.; Ukon, R.; Itoh, R. J. Org. Chem. 2005, 70, 6282-6290. (e) Kawamura, T.; Inagaki, F.; Narita, S.; Takahashi, Y.; Hirata, S.; Kitagaki, S.; Mukai, C. Chem. Eur. J. 2010, 16, 5173-5183.

19. From 17, the used of acetic anhydride in the presence of a catalytic amount of $\mathrm{Cu}(\mathrm{OTf})_{2}$ led to $\mathbf{2 1}$ in a moderate $58 \%$ yield. 
Chandra, K.; Saravanan, P.; Singh, V. K. Tetrahedron Lett. 2001, 42, 5309-5311. 